\title{
Fibrilación auricular posoperatoria ¿Un lobo con piel de cordero?
}

\author{
Dr. Jorge Estigarribia Passaro
}

\begin{abstract}
Resumen ratorio, y no es esperable su abatimiento en el futuro cercano. cuanto su evolución y manejo. lizar las medidas preventivas. entorno del perioperatorio.

Palabras clave: CIRUGÍA CARDÍACA

POSOPERATORIO

FIBRILACIÓN AURICULAR

REVISIÓN
\end{abstract}

Desde época temprana de la cirugía cardíaca, la fibrilación auricular (FA) ha sido un acompañante frecuente del posope-

La interpretación de su significado clínico se ha modificado en los últimos años, una vez conocidas su tendencia recurrente y su asociación con serias complicaciones inmediatas y a largo plazo.

Este hecho deja entrever un nuevo desafío, ya que ha dejado de ser un problema menor y de consideración puntual en el perioperatorio para constituir un tema de preocupación y seguimiento en el futuro alejado, aún con incertidumbres en

La profilaxis efectiva de esta arritmia se ha dificultado por la multiplicidad de factores de riesgo y lo intrincado de su génesis, sumado a la edad creciente de los pacientes intervenidos, la complejidad mayor de los procedimientos, los posibles efectos colaterales de los fármacos empleados y la inexistencia de un algoritmo predictivo confiable que permita raciona-

Además, muchas recomendaciones de las guías de práctica clínica actuales se basan en información obtenida en estudios realizados en la FA primaria, por lo que su adopción en el escenario de la cirugía cardíaca ha sido menor a la deseable.

Todos estos aspectos son objeto de análisis en esta revisión, que finaliza con pautas de manejo práctico de la arritmia en el

\section{Postoperative atrial fibrillation: a wolf with lamb skin?}

\section{Summary}

Since an early age of heart surgery, atrial fibrillation has been a frequent companion of the postoperative period, and its decline is not to be expected in the near future.

The interpretation of its clinical significance has changed in recent years, after knowing its recurrent trend and its association with serious immediate and long-term complications.

This fact unveils a new challenge, as it is no longer a minor problem of consideration restricted to the perioperative period and has become a topic of concern and follow-up in the distant future, still with uncertainties as to its evolution and management. The effective prophylaxis of this arrhythmia has been difficult by the multiplicity of risk factors and the intricate of its genesis, added to the increasing age of the patients involved, the greater complexity of the procedures, the possible side effects of the drugs used and the absence of a reliable predictive algorithm that could allow to rationalizing preventive measures. In addition, many recommendations from current clinical practice guidelines are based on information obtained from studies in primary atrial fibrillation, so their adoption in the heart surgery scenario has been less than desirable.

All these aspects are analyzed in this review, which ends with directives for the practical management of the arrhythmia in the perioperative environment.

Key words: $\quad$ CARDIAC SURGERY

POSTOPERATIVE

ATRIAL FIBRILLATION

REVIEW

Instituto Nacional de Cirugía Cardíaca (INCC). Montevideo, Uruguay.

Correspondencia: Dr. Jorge Estigarribia Passaro. Correo electrónico: jorgeestigarribia@movinet.com.uy

El autor declara no tener conflictos de intereses.

Recibido Oct 12, 2020; aceptado Oct 20, 2020 


\section{Fibrilação auricular pós-operatória: ¿um lobo com pele de cordeiro?}

\section{Resumo}

Desde os primeiros días da cirurgia cardíaca, a fibrilação atrial (FA) tem sido uma companheira frequente para o pós-operatório, e sua reduçao não é esperada em um futuro próximo.

A interpretação de sua significância clínica mudou nos últimos anos, tendo conhecido sua tendência recorrente e sua associação com sérias complicações imediatas e de longo prazo.

Este fato mostra um novo desafio, pois deixou de ser um pequeno problema e uma consideração oportuna no perioperatório para constituir um tema de preocupação e acompanhamento em um futuro distante, mesmo com incertezas quanto à sua evolução e gestão.

A profilaxia efetiva dessa arritmia tem sido cercada pela multiplicidade de fatores de risco e pela intrincação de sua gênese, juntamente com a idade crescente dos pacientes envolvidos, a maior complexidade dos procedimentos, os possíveis efeitos colaterais dos medicamentos utilizados e a ausência de um algoritmo preditivo confiável para racionalizar as medidas preventivas.

Além disso, muitas recomendações das guias atuais de prática clínica são baseadas em informações obtidas em estudos conduzidos em FA primária, de modo que sua adoção no cenário da cirurgia cardíaca tem sido menos do que desejável.

Todos esses aspectos são analisados nesta revisão, que termina com diretrizes práticas de gestão para arritmia no ambiente perioperatório.

$\begin{array}{ll}\text { Palavras-chave: } & \text { CIRURGIA CARDÍACA } \\ & \text { PÓS-OPERATÓRIO } \\ & \text { FIBRILAÇÃO ATRIAL } \\ & \text { REVISÃO }\end{array}$

"Cuando el peligro nos parece leve, deja de ser leve". Francis Bacon. Filósofo, político, abogado y escritor inglés

(1561-1626)

\section{Introducción}

La fibrilación auricular (FA) es la arritmia más común en el posoperatorio de cirugía cardíaca (POCC) y probablemente la complicación de mayor frecuencia en ese contexto. Considerada inicialmente un suceso benigno, se la ha asociado más recientemente a serias complicaciones con impacto pronóstico a mediano y largo plazo, mayor consumo de recursos materiales y humanos, y excesivos costos de la atención sanitaria. Si tal asociación es un efecto propio de la arritmia o esta es solo un marcador de mayor deterioro miocárdico y peores condiciones comórbidas con consecuencias alejadas, es motivo de persistente discusión.

Esta relación adversa estimuló el interés en precisar sus factores de riesgo y mecanismos de producción para desarrollar medidas costo-efectivas de profilaxis, aunque su multicausalidad y complejidad fisiopatológica condujeron a una diversidad de propuestas con escasa repercusión en su incidencia. El aumento progresivo de la edad y la prevalencia creciente de comorbilidades en los pacientes sometidos a procedimientos cardioquirúrgicos es una explicación probable de este hecho.

La presente revisión provee una mirada global del tema, con consideraciones sobre epidemiología, mecanismos de producción, repercusión clínica, medidas preventivas y manejo inmediato de esta complicación, proponiendo además una perspectiva de lo que a la luz del conocimiento actual debería representar para el clínico, más allá del episodio agudo.

\section{Definición e incidencia}

La FA posoperatoria (FAPO) es la que aparece de novo en este contexto. Es más frecuente en el POCC que luego de cirugía torácica no cardíaca, y francamente menor después de cirugía no torácica ${ }^{(1)}$. Su incidencia depende del criterio diagnóstico, referido al umbral de duración y/o reiteración requerido para su consideración, el método de registro, las características de la población intervenida, y el horizonte temporal definido para su notificación. Dada la marcada variabilidad en estos aspectos, las cifras más citadas de incidencia se sitúan entre $20 \%$ y $40 \%$, aunque con extremos entre $15 \%$ y $60 \%$ en poblaciones seleccionadas.

Pese a la implementación de diferentes estrategias de prevención, la FAPO no muestra una tendencia decreciente en el curso de las décadas ${ }^{(2,3)}$, dado que se relaciona fuertemente con la edad y con alteraciones estructurales y de la carga hemodinámica del miocardio auricular que acompañan a comorbilidades como insuficiencia cardíaca, diabetes, obesidad, nefropatía, enfermedad pulmonar obstructiva crónica, etcétera. Tanto la edad como la prevalencia de estas patologías, estrechamente interrelacionadas, han experimentado un incremento lento pero continuo en la población que recibe cirugía cardíaca. 


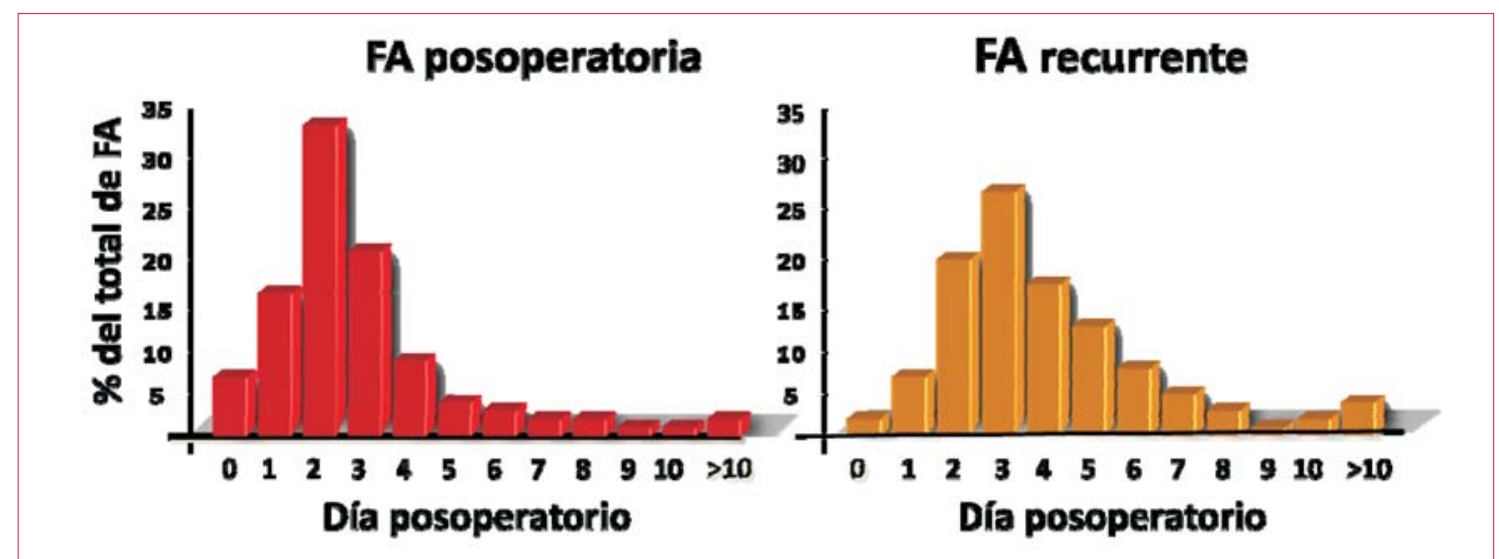

Figura 1. Distribución diaria típica de la incidencia de FAPO. Modificado de Mathew y colaboradores ${ }^{(8)}$.

El tipo y la complejidad del procedimiento quirúrgico influyen significativamente; su incidencia es relativamente menor en la revascularización miocárdica aislada, con cifras crecientes para sustitución valvular aórtica, sustitución valvular mitral, y asociación de revascularización con sustitución valvular, en ese orden ${ }^{(4)}$. La menor incidencia ocurre luego del trasplante cardíaco ortotópico, dado que las aurículas trasplantadas quedan desconectadas eléctricamente de las venas pulmonares y, producto de la denervación, sin influencia del sistema neurovegetativo(5), aunque se ha comprobado que el rechazo del órgano se vincula significativamente con taquiarritmias auriculares tardías ${ }^{(6)}$. La mayoría de los episodios se acumulan dentro de la primera semana del posoperatorio $(\mathrm{PO})^{(7)}$. Al cuarto día de la cirugía se presentan el 74\% de los episodios, y al sexto el $94 \%$. La incidencia pico del primero se sitúa típicamente en el segundo día, y la primera recurrencia en el tercer día(8) (figura 1), lo que proporciona una ventana de tiempo considerable para implementar medidas de profilaxis.

\section{Factores de riesgo}

La lista de condiciones que se han asociado a FAPO es muy extensa, aunque con escasa reproducibilidad, lo que dificulta identificar un blanco concreto con validez generalizada para su profilaxis.

Conceptualmente, agruparemos a los factores asociados a FAPO en dos tipos de sustrato, ubicados en diferente contexto temporal. Existe un sustrato basal o vulnerable, representado por el grado de remodelación auricular con el que el paciente llega a la cirugía, relacionado con la edad y diversas condiciones patológicas ${ }^{(9)}$. Esta condición, que se ha catalogado como una "miopatía atrial" ${ }^{(10,11)}$ determina qué tan cerca o tan lejos se sitúa el paciente de lo que podríamos considerar el umbral de FA. El segundo es el sustrato facilitador: modificaciones desencade- nadas por la cirugía y sus técnicas de soporte (anestesia, circulación extracorpórea [CEC], fármacos, etcétera), que inciden sobre la actividad eléctrica auricular a través varias vías fisiopatológicas.

La interacción entre ambos sustratos definirá si se alcanza o no dicho umbral ${ }^{(12)}$.

Estas vías son la inflamación local y general, la hiperactividad simpático-adrenal, la injuria por isquemia/reperfusión/estrés oxidativo y alteraciones hidroelectrolíticas (figura 2), fenómenos propios del ambiente posoperatorio y frecuentemente autolimitados en el tiempo. A pesar de esta máscara de transitoriedad, la FAPO se asocia con una posibilidad hasta ocho veces mayor de presentar FA en el futuro alejado ${ }^{(13)}$, señalando la importancia del deterioro previo del miocardio auricular.

Algunos factores intraoperatorios incrementan la incidencia de FAPO mediante la activación de los mecanismos fisiopatológicos mencionados: tiempos de clampeo y CEC prolongados, mala protección miocárdica, incisiones atriales, canulación venosa bicava y colocación de un vent (aspirador) en el ventrículo izquierdo a través de venas pulmonares. La cirugía sin CEC parece tener un papel protector, pero algunas experiencias no apoyan este concepto.

En la figura 3 se muestra la compleja interrelación entre alteraciones de diversas variables fisiológicas en el intra y el PO y las grandes vías patogénicas que desencadenan la arritmia.

Se ha intentado cuantificar el riesgo de FAPO mediante la elaboración de sistemas de puntuación o scores, para identificar preoperatoriamente a los pacientes de mayor riesgo y aplicar medidas profilácticas dirigidas y más eficaces. Sin embargo, un análisis pos-hoc de validación utilizando tres scores derivados de algunos de los estudios de mayor extensión obtuvo un bajo valor predictivo cuando se aplicó en una cohorte prospectiva de validación ${ }^{(15)}$. 


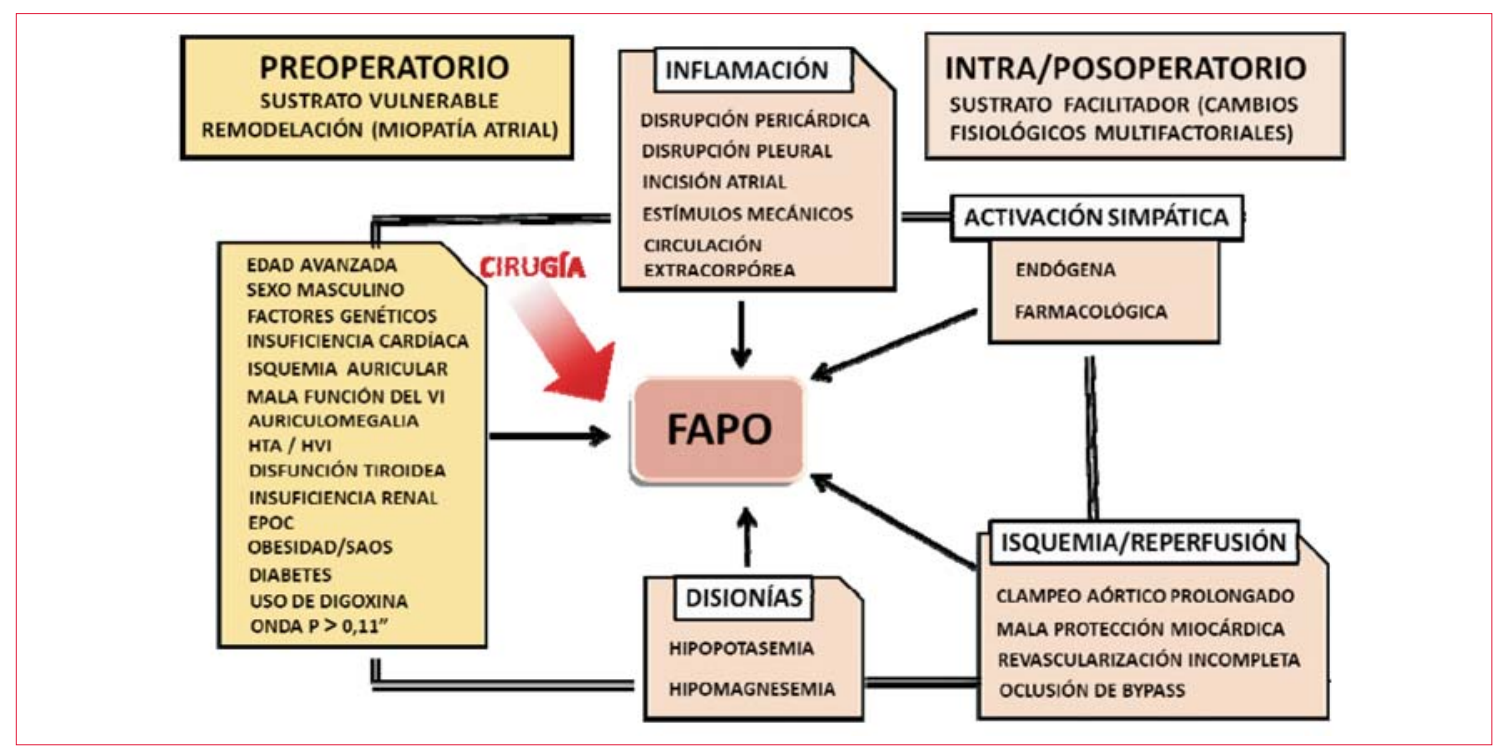

Figura 2. Factores de riesgo de fibrilación auricular posoperatoria. HTA: hipertensión arterial; HVI: hipertrofia ventricular izquierda; SAOS: síndrome de apnea obstructiva del sueño.

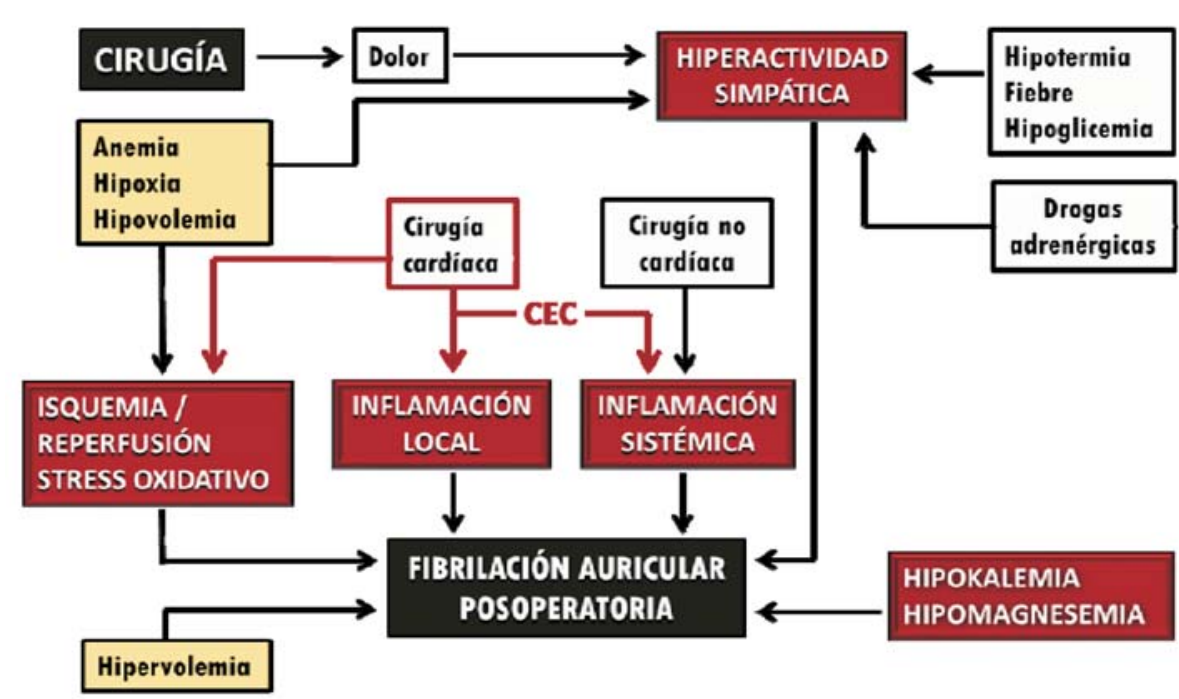

Figura 3. Fisiopatología de la fibrilación auricular posoperatoria. La alteración de diversas variables fisiológicas por la cirugía y el posoperatorio, activa cuatro vías principales con acción directa sobre las propiedades físicas y eléctricas del miocardio auricular. Estas desviaciones fisiológicas son a la vez adecuados blancos terapéuticos a atacar con medidas farmacológicas y no farmacológicas en una estrategia integral de profilaxis. CEC: circulación extracorpórea. (Adaptado de Chelazzi y colaboradores $\left.{ }^{(14)}\right)$

\section{Mecanismos básicos de inicio y persistencia}

La patogénesis de la FAPO, como la que acontece fuera del contexto quirúrgico, se apoya en una hipótesis dual (figura 4). La fibrosis y la disminución de la velocidad de conducción y/o del período refractario efectivo en algunas áreas del miocardio auricular generan un bloqueo funcional, acentuado por las alteraciones propias de la cirugía y el PO. En estas condiciones, el evento gatillo, representado por varios estímulos prematuros en sucesión, da inicio a múltiples circuitos de reentrada simultáneos que colisionan en el miocardio atrial, manteniendo una conducción aleatoria ${ }^{(16)}$, con pasaje irregular a través del nodo aurículoventricular. Estas descargas ectópicas se originan con frecuencia en fibras miocárdicas auriculares que penetran algunos centímetros en las paredes de las venas pulmonares $^{(17)}$ y manifiestan un automatismo exacerbado ante desequilibrios neurovegetativos o la distensión auricular.

Menos frecuentemente, el foco se ubica en la base del apéndice auricular izquierdo, la desembocadura de la vena cava superior o el ligamento de Marshall. 


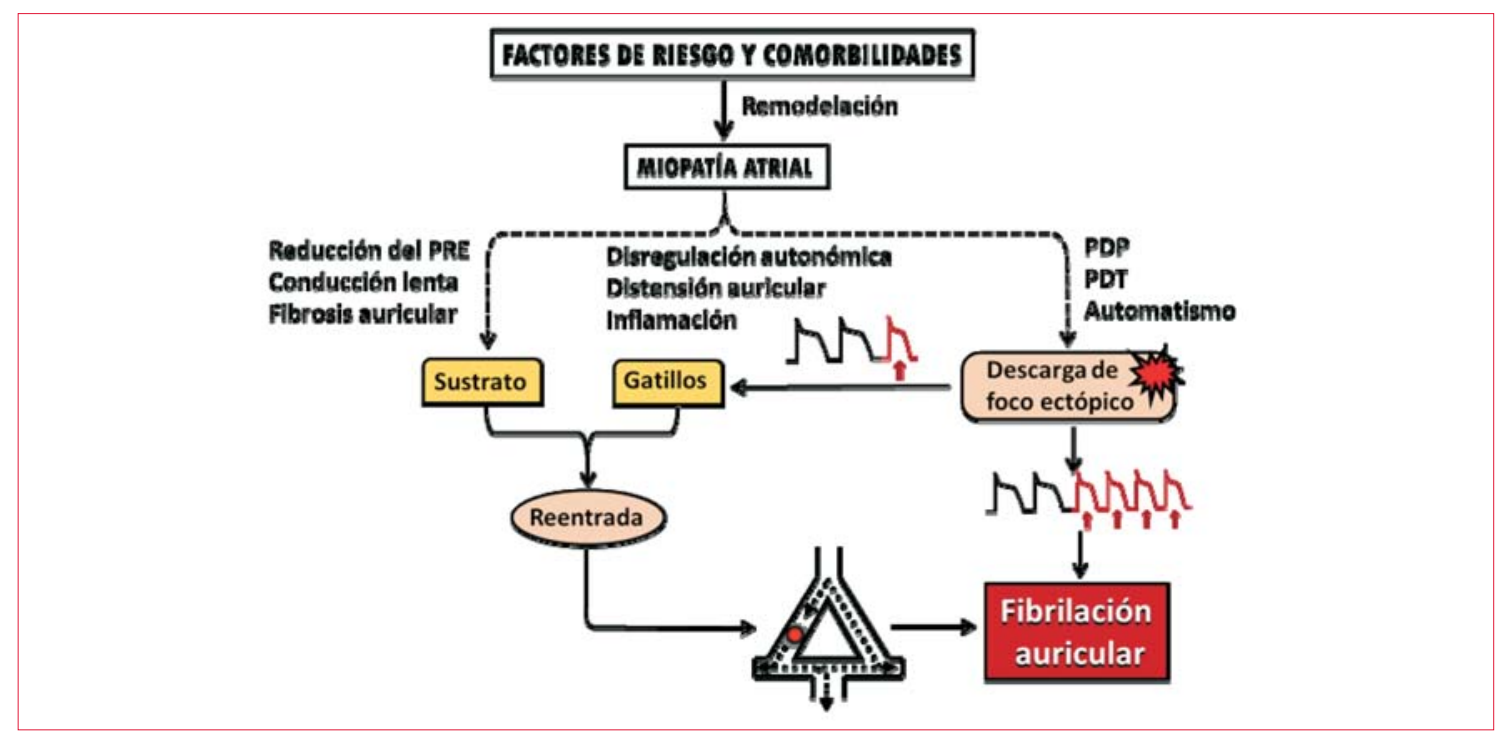

Figura 4. Mecanismos electrofisiológicos de la fibrilación auricular posoperatoria. La edad avanzada y múltiples condiciones patológicas tanto cardiovasculares como extracardíacas inducen una miopatía atrial por remodelación funcional y estructural. Esta constituye el sustrato electrofisiológico vulnerable a la fibrilación auricular posoperatoria, sustentado en fibrosis, reducción del período refractario efectivo (PRE) y de la velocidad de conducción. Los eventos gatillo (firing) consisten en descargas ectópicas focales rápidas originadas en pos-despolarizaciones precoces (PDP) o tardías (PDT), frecuentemente iniciadas en la desembocadura de las venas pulmonares, que desencadenan bloqueo unidireccional y reentrada. Ambos sustratos son favorecidos por las alteraciones fisiológicas del posoperatorio. La hiperactividad sostenida del foco responsable puede ser un factor de persistencia de la arritmia, y la remodelación atrial adicional que esta genera, obra en el mismo sentido. PRE: período refractario efectivo; PDP: pos-despolarizaciones precoces; PDT: posdespolarizaciones tardías. (Modificado de Nattel y colaboradores ${ }^{(18)}$ ).

La propia FA acentúa la remodelación tiempo-dependiente de la cámara por reducción adicional del período refractario efectivo y dilatación auricular. La continuidad y/o recurrencia de la arritmia dependerán del grado de remodelación electromecánica atrial, la evolución del contexto arritmogénico PO y la eficacia del tratamiento adoptado.

\section{Impacto clínico}

La FAPO fue inicialmente contemplada como una complicación autolimitada y benigna, quizás porque su estudio se limitó al período perioperatorio. Sin embargo es hoy innegable su asociación con serios eventos adversos y mortalidad, incluso en un horizonte temporal amplio.

Los efectos clínicos de la FAPO dependen de la condición cardiorrespiratoria previa del paciente, la persistencia y/o recurrencia de los episodios arrítmicos y la frecuencia cardíaca alcanzada.

Habitualmente se presenta en episodios breves, paroxísticos, a menudo asintomáticos, con tendencia a la remisión espontánea y recurrencias dentro de la primera semana.

Es frecuente una sensación inespecífica de inquietud, desasosiego o disconfort torácico, en ocasiones acompañada de palpitaciones rápidas e irregulares o latidos cervicales.
En presencia de disfunción o hipertrofia del ventrículo izquierdo, condiciones de sobrecarga de volumen, o en caso de isquemia residual, pueden presentarse ángor, hipotensión arterial, disnea o incluso edema pulmonar franco o shock. Estas diferentes formas de expresión clínica condicionan la oportunidad, el objetivo y la naturaleza de la respuesta terapéutica.

La FAPO ha sido crecientemente asociada con severas complicaciones en diferentes sistemas fisiológicos, reingreso a la unidad de cuidados intensivos (UCI), reintubación, internación posoperatoria prolongada, infecciones, reintervención por sangrado, peor calidad de vida y mayor mortalidad precoz y tardía ${ }^{(19,20)}$, aunque sin unanimidad entre los estudios, dada la variabilidad en múltiples aspectos de su diseño. Esta asociación no implica necesariamente una relación de causalidad, y con la prevención efectiva de la arritmia no siempre se demuestra una reducción de dichas complicaciones, ni del tiempo de internación. Más aún, la relación causa-efecto puede ser inversa en muchos de estos eventos.

\section{Recurrencias de fibrilación auricular y ataque cerebrovascular}

Una de las más temidas complicaciones asociadas con la FAPO es el ataque cerebrovascular (ACV) isquémico o su variante menor, el ataque isquémico 
transitorio (AIT), ya señalados como complicaciones precoces en algunos estudios iniciales ${ }^{(19,21)}$. Su abatimiento se presenta como uno de los objetivos centrales en las estrategias de profilaxis de la FAPO y del tratamiento anticoagulante. Sin embargo, en algunos estudios no se comprueba una asociación independiente entre FAPO y $\mathrm{ACV}^{(8,22-24)}$, proponiéndose mecanismos alternativos de producción. El empleo de una definición restrictiva de FAPO y/o la anticoagulación prolongada podrían explicar este resultado.

Conocer el potencial embolígeno precoz y a largo plazo de la FAPO define la estrategia de tratamiento anticoagulante y su alcance temporal. En cirugía valvular, la frecuente indicación de anticoagulación prolongada puede conducir a subestimar el riesgo embolígeno futuro de la FA, e inversamente, la presentación de un ACV puede deberse a una embolia de origen protésico. Por este motivo, los estudios se han enfocado particularmente en la cirugía de revascularización miocárdica.

Loubani y colaboradores ${ }^{(25)}$, mostraron en su serie de 375 pacientes revascularizados que del $25 \%$ que presentó FAPO, la mitad persistía en FA al alta sanatorial y $39 \%$ a los 6 meses, sin diferencias en la incidencia de ACV.

Cioffi y colaboradores ${ }^{(26)}$ observaron que la recidiva de algún episodio de FA a los 30 días fue de $50 \%$ sin profilaxis antiarrítmica luego del alta, $47 \%$ con dosis modestas de amiodarona y $10 \%$ con betabloqueantes $(p=0,002)$, aunque a los 10 meses no resultó frecuente su reiteración.

La irrupción de uno o más episodios de FAPO en pacientes con ritmo sinusal recuperado al momento del alta no solo predice FA a largo plazo, sino también ACV alejado.

Gialdini y colaboradores ${ }^{(27)}$ encontraron, con un seguimiento medio de 2,1 años, que la incidencia de FA luego de cirugía cardíaca fue 4,8 veces mayor entre pacientes que presentaron FAPO, y que a un año se asoció en forma independiente con ACV, y más fuertemente aun considerando específicamente el ACV embólico, el que además guardó una relación directa con el score $\mathrm{CHA}_{2} \mathrm{DS}_{2}$-VASc.

En un metaanálisis con ocho estudios y 1.157 pacientes monitorizados hasta dos años del PO con diferentes métodos, la recurrencia de FA fue de $28,3 \%$ a las cuatro semanas. En dos de los estudios con monitorización continua mediante dispositivos implantados, la FA se presentó en el $61 \%$ y en $100 \%$ a los dos años. El $40 \%$ y el $93 \%$ de los episodios arrítmicos respectivamente, fueron asintomáticos ${ }^{(28)}$.

En otro metaanálisis con 35 estudios y más de 2.450.000 pacientes, la FAPO incrementó en un
$62 \%$ el ACV precoz y un $37 \%$ en un seguimiento entre uno y 17,8 años ${ }^{(29)}$.

Megens y colaboradores ${ }^{(30)}$ en su metaanálisis de 16 estudios con 108.711 pacientes revascularizados seguidos durante dos años, también observaron, utilizando un minucioso análisis estadístico, que la FAPO se asoció con un aumento significativo del riesgo de ACV en los estudios ajustados .

La misma conclusión se desprende del metaanálisis recientemente publicado de Kerwin y colaboradores, con 19 estudios y 129.628 pacientes revascularizados, y la mayoría de estudios reportando el evento a $>1$ año( ${ }^{(31)}$.

Resulta entonces evidente la elevada incidencia de FA recurrente luego de FAPO y un importante riesgo oculto de fenómenos embólicos a largo plazo, apoyando la eventual necesidad de un tratamiento anticoagulante prolongado o al menos un seguimiento minucioso del ritmo cardíaco por tiempo indefinido. El concepto de miopatía atrial, retomado y profundizado recientemente por Shen y colaboradores $^{(11)}$ puede representar un abordaje adecuado para valorar el riesgo de ACV con independencia del perfil evolutivo de la FA y refinar el criterio para iniciar y mantener la anticoagulación.

\section{Deterioro neurocognitivo}

Algunos informes señalan que aún en ausencia de daño neurológico estructural, utilizando diferentes instrumentos de evaluación, los pacientes que experimentan uno o varios episodios de FAPO muestran un deterioro cognitivo significativo a las seis semanas del $\mathrm{PO}^{(8,32)}$.

Considerando el perfil etario actual del paciente candidato a cirugía cardíaca, en quien no son raros los antecedentes de enfermedad cerebrovascular y/o de afectación cognitiva preoperatoria, estos datos suman un argumento para redoblar esfuerzos en la prevención de esta arritmia.

\section{Mortalidad}

Resulta del mayor interés clínico definir si la FAPO está asociada a mortalidad hospitalaria y/o alejada, y si así fuera, si es un efecto independiente de la arritmia o esta es un marcador de menor sobrevida por efecto de covariables. Este objetivo se ha dificultado por el diseño retrospectivo de gran parte de los estudios, la posibilidad de una incompleta consideración de factores de confusión en el análisis multivariado, e inconvenientes para lograr cohortes realmente equilibradas en los estudios con muestras pareadas $^{(23)}$.

Además, inconsistencias en la definición de FAPO, como la que solo considera la FA que requie- 
re tratamiento, pueden conducir a una subestimación de su prevalencia y de la mortalidad asociada.

El estudio original de Almassi y colaboradores fue de los primeros en mostrar la FAPO como un evento con serio impacto pronóstico a corto y mediano plazo, duplicando la mortalidad tanto hospitalaria como a los seis meses, además de una incidencia significativamente mayor de varios eventos adversos: readmisión a UCI, reintubación, infarto agudo de miocardio (IAM), insuficiencia cardíaca congestiva (ICC) y $\mathrm{ACV}^{(21)}$.

Sin embargo, el mismo autor en un estudio reciente, solo reportó una tendencia no significativa a mayor mortalidad a cinco años, aunque utilizando un intervalo de confianza más exigente ${ }^{(24)}$.

En el ya referido estudio de Loubani y colaboradores $^{(25)}$ no se demostró asociación de la FAPO con eventos adversos, pero la población estudiada era pequeña y el seguimiento limitado.

Villareal y colaboradores ${ }^{(33)}$ informaron una mortalidad hospitalaria y a cuatro o cinco años significativamente mayor en pacientes con FAPO, tanto en el análisis de regresión logística de su cohorte retrospectiva, como en el subgrupo de casos pareados.

Tulla y colaboradores ${ }^{(23)}$ compararon dos cohortes de 139 pacientes emparejadas mediante propensity score matching; una con FAPO persistente al momento del alta, y otra sin FAPO (grupo RS). Al término de 8,5 años, 28,3\% del grupo FAPO estaba en FA crónica y sufrió una mortalidad global del $33,3 \%$ y cardíaca del $15,2 \%$, mientras que las cifras para el grupo RS fueron $5,1 \%, 18,8 \%$ y $4,3 \%$ respectivamente ( $\mathrm{p}<0,002$ para todas las comparaciones). La incidencia de ACV fue similar, pero todos los pacientes con FA fueron anticoagulados a largo plazo.

Varios metaanálisis apoyan la incidencia adversa de la FAPO en la mortalidad precoz y alejada en el POCC: el de Kaw y colaboradores, que incluye 40.112 pacientes, con efecto hasta cuatro años ${ }^{(34)}$, el ya referido de Meng-Hsin Lin ${ }^{(29)}$ con 2.458 .010 pacientes, (ambos en cirugía coronaria y combinada), y el también mencionado de Kerwin ${ }^{(31)}$ con 129.620 individuos que recibieron cirugía de revascularización miocárdica aislada.

La presunción de causalidad entre FAPO y mortalidad es reforzada por el análisis de la mortalidad causa-específica, la intervención de un mecanismo plausible y la comprobación de que el tratamiento de ese mecanismo es capaz de reducir el evento.

El-Chami y colaboradores estudiaron 16.169 pacientes revascularizados empleando un minucioso análisis multivariado. Observaron una mortalidad a seis años significativamente mayor en pacientes con FAPO, excluyendo la mortalidad hospitalaria. Los pacientes que recibieron el alta bajo tratamiento con warfarina presentaron menor mortalidad en el seguimiento, planteándose un probable mecanismo embólico del deceso ${ }^{(35)}$.

El estudio prospectivo de Mariscalco y colaboradores en cirugía de bypass coronario $(\mathrm{n}=1.832)$ con seguimiento medio de 5,1 años, mostró una mayor mortalidad tanto hospitalaria como a largo plazo. Es destacable que la única causa de muerte que resultó significativa fue la embólica(36).

Ahlsson y colaboradores ${ }^{(13)}$ en su seguimiento a seis años de 571 pacientes revascularizados observaron que la FAPO resultó el más potente predictor independiente de FA futura, y documentaron una mortalidad de $29,7 \%$ vs. $14,8 \%$ en pacientes con y sin FAPO respectivamente $(\mathrm{p}<0,001)$. La muerte de causa cerebral isquémica fue mucho más común entre los primeros (4,2\% vs. $0,2 \%$; p < 0,001).

Otro estudio con 6.821 pacientes revascularizados seguidos durante 9,8 años reveló también una interacción independiente de la FAPO con mortalidad alejada, y que la mayor causa de muerte ajustada estuvo relacionada con arritmia, a la que se suma la enfermedad cerebrovascular ${ }^{(37)}$.

Estos datos, acordes al concepto bien consolidado de que la FA constituye un desorden progresivo ${ }^{(38,39)}$, sugieren que la FAPO luego de cirugía de revascularización miocárdica y/o la miopatía auricular subyacente comportan una causa propia de ACV y mortalidad alejados, básicamente por ser precursores de una etapa más estable, frecuentemente silenciosa y de mayor potencial embolígeno de la arritmia (figura 5), y no representan simples subrogantes de un grado más evolucionado de enfermedad cardíaca, aunque este mayor deterioro sea también comprobable y genere su propia cuota de mortalidad.

\section{Efecto sobre el flujo de los injertos coronarios}

La FA puede comprometer considerablemente el gasto cardíaco y la perfusión de diversos órganos, incluido el propio corazón, donde al deterioro de la perfusión se suma el incremento del consumo de $\mathrm{O}_{2}$ debido al aumento de la frecuencia cardíaca, agravando el disbalance entre oferta y demanda.

Varios estudios han señalado una incidencia significativamente mayor de IAM PO en pacientes que experimentan $\mathrm{FAPO}^{(21,22)}$. Si bien el IAM es una causa posible de esta arritmia, también la FA puede favorecer la complicación isquémica en un paciente recientemente revascularizado.

$\mathrm{Al}$ respecto resulta interesante el estudio de Shin y colaboradores, en el que durante un episodio provocado de FA al momento de la revisión de los 


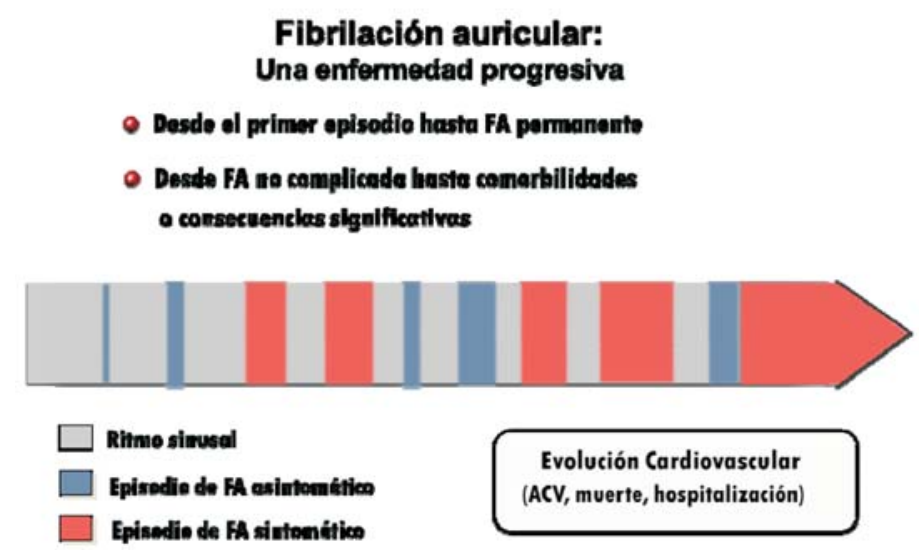

Figura 5. Progresión de la fibrilación auricular. La remodelación eléctrica, mecánica y estructural de la fibrilación auricular es un proceso dinámico y autopromovido que se traduce en manifestaciones clínicas y complicaciones progresivas. FA: fibrilación auricular; ACV: ataque cerebrovascular.

puentes, además de un deterioro hemodinámico apreciable se produce un descenso significativo de su flujo, más acusado en el caso de la arteria mamaria interna por su mayor dependencia del período diastólico ${ }^{(40)}$.

\section{Implante de marcapasos definitivo}

Se ha informado un incremento significativo del requerimiento de marcapasos definitivo en pacientes con FAPO, sea en el PO inmediato ${ }^{(19)}$, o en el seguimiento alejado ${ }^{(23)}$. El uso de fármacos con efecto crono y dromotrópico negativo en el manejo de los episodios de FA puede ser una explicación parcial, pero también participa un deterioro funcional de los nodos sinusal y aurículoventricular como expresión de la remodelación auricular propia de la arritmia ${ }^{(41)}$.

\section{Calidad de vida}

En el estudio de Bramer y colaboradores, cuatro de 10 scores de calidad de vida puntuaron peor luego de cirugía de bypass coronario en pacientes con FAPO. Además, esta fue un predictor negativo independiente de recuperación de la calidad de vida a los seis meses del PO, tanto en los componentes físicos como mentales ${ }^{(42)}$.

\section{Duración de la internación y costos}

Casi invariablemente se ha vinculado a la FAPO con mayor tiempo de internación en UCI y hospitalaria total, con incrementos en torno a 36-48 horas y tres o cuatro días respectivamente $\mathrm{e}^{(13,19,21,22,33,43)}$. Si bien pueden existir dificultades en el control de la arritmia y en la obtención de una anticoagulación efectiva, la causa determinante son las múltiples complicaciones con las que se asocia. Paralelamente, la FAPO representa un sustancial exceso de costos evaluados en diferentes tipos de procedimien- to ${ }^{(44,45)}$. Este costo excedente parece mantenerse a un año, aunque no entre los dos a cinco años ${ }^{(24)}$.

\section{Profilaxis}

Pese a la disponibilidad de varias guías de práctica clínica con propuestas de profilaxis fundamentadas, existe una limitada adherencia a recomendaciones de clase I y IIa. Esta brecha de implementación es probablemente debida a una percepción desfavorable de la relación riesgo-beneficio de los fármacos disponibles.

La prevención efectiva de la FA no supone necesariamente que los eventos adversos asociados disminuyan correlativamente, por tres posibles razones: a) que el suceso estudiado no sea causado por la arritmia, sino simplemente una covariable, o incluso que la FA sea consecuencia de aquel, por ejemplo: infección, ICC, IAM; b) que el evento tenga una baja incidencia y un mecanismo alternativo de producción, como ocurre con el ACV; y c) que los efectos colaterales de los fármacos utilizados en su profilaxis favorezcan el evento y anulen un posible beneficio: bradicardia severa, ICC, implante de MPD, reingreso a UCI.

Por otra parte, la multicausalidad de esta arritmia y la interacción entre sus mecanismos patogénicos (figura 3) ofrecen la oportunidad de implementar medidas no farmacológicas sobre distintos aspectos del proceso asistencial.

De acuerdo a un principio general, el rendimiento de las medidas profilácticas será tanto mayor cuanto más probable es el evento a prevenir, por lo que se impone una racionalización de las acciones acorde a la valoración del riesgo. 


\section{Betabloqueantes}

Son los fármacos más estudiados y gozan de acuerdo general en cuanto a beneficio en la profilaxis de la FAPO, con una recomendación clase I A o I B según las diferentes guías en pacientes con riesgo normal o alto de FAPO y en ausencia de contraindicaciones $^{(46-49)}$.

Sin embargo, entre el 30\% y el $50 \%$ de los médicos encuestados en Europa y en EEUU no los utiliza rutinariamente en pacientes de alto riesgo ${ }^{(8,52)}$.

Determinan una reducción de la FAPO en torno al $70 \%{ }^{(50)}$, que desciende a un $31 \%$ (aún significativa) considerando solo los estudios en los que no se requiere la suspensión de los betabloqueantes fuera de protocolo por parte de los controles ${ }^{(51)}$. Son efectivos iniciándolos desde el preoperatorio o en el PO inmediato. Su beneficio se relaciona con la atenuación del estado de hipertonía simpática habitual de este contexto, con efecto tanto sobre el foco gatillo como en la reentrada. No han demostrado un efecto significativo sobre la duración de la internación, el ACV o la mortalidad ${ }^{(52)}$. Se han utilizado distintos fármacos, incluyendo propranolol, atenolol, nadolol, metoprolol y carvedilol.

El sotalol, con propiedades antiarrítmicas de clase III, mostró un mayor efecto comparativo, pero también mayor incidencia de ritmos lentos, hipotensión, proarritmia y necesidad de suspensión, por lo cual se le asigna una indicación IIb $^{(48)}$.

La suspensión de los betabloqueantes previo a la cirugía es contraproducente, ya que se asocia a un incremento de la incidencia de la FA por sobrerregulación de receptores $ß$.

\section{Amiodarona}

Este antiarrítmico de clase III es también bloqueador de los canales de sodio (I), de los receptores beta-adrenérgicos (II) y antagonista del calcio (IV).

Su toxicidad pulmonar, hepática, tiroidea, cutánea y corneal es rara a corto plazo, pero su uso intrahospitalario I/V puede generar bradicardia e hipotensión. Tiene menor potencial proarrítmico que los demás fármacos de clase III, incluido el sotalol.

En el estudio randomizado PAPABEAR ${ }^{(53)}$ la amiodarona oral durante seis días antes y seis días luego de cirugía cardíaca de coordinación mostró una reducción global de la incidencia de taquiarritmias auriculares en distintos subgrupos, asociada o no a betabloqueantes, sin modificar la mortalidad ni los reingresos. Un metaanálisis demostró que las dosis totales medias ( 3 a $5 \mathrm{~g}$ ) son tan efectivas como las altas ( $>5 \mathrm{~g}$ ) y que es igualmente efectiva si se comienza en el PO inmediato por vía I/ $\mathrm{V}^{(54)}$.

En el metaanálisis de Cochrane de 33 estudios redujo la incidencia de FAPO, y a diferencia de los betabloqueantes, también descendió la duración de la internación, aunque no la mortalidad(50).

En nuestro medio, un estudio no randomizado mostró menor incidencia acumulada de FA en el grupo con profilaxis completa según protocolo, pero el grupo control tenía mayor riesgo basal de presentar la arritmia ${ }^{(55)}$.

La amiodarona recibe una recomendación de clase I A en equivalencia a los betabloqueantes en la guía de la European Society of Cardiology (ESC) $2020^{(49)}$, mientras que su versión previa (2016) y en las restantes, es clase IIa. En pacientes de riesgo medio o moderado, puede utilizarse en sustitución de los betabloqueantes cuando están contraindicados. La asociación de ambos logra una mayor reducción de la FAPO que los betabloqueantes aisla$\operatorname{dos}^{(56)}$. Esta opción es atractiva en pacientes con riesgo elevado de la arritmia, aunque requiere una indicación personalizada y estricta vigilancia por la posible potenciación de sus efectos indeseables.

\section{Estatinas}

ARMYDA-3 fue el primer estudio randomizado que mostró una reducción significativa de la FAPO en cirugía cardíaca de coordinación utilizando una estatina desde los 7 días previos a la operación. La duración de la internación fue levemente menor, pero los eventos mayores no se diferenciaron ${ }^{(57)}$.

Estudios posteriores en cirugía de bypass coronario o valvular, mostraron resultados conflictivos. La mayoría de los metaanálisis confirmó un efecto protector contra la arritmia. El de Kuhn y colaboradores, con 54 estudios y más de 90.000 pacientes concluyó que el uso preoperatorio de estatinas no solo redujo la incidencia de FAPO, sino también de ACV, mortalidad total y tiempo de internación ${ }^{(58)}$.

Otro metaanálisis encontró que atorvastatina fue eficaz, pero no rosuvastatina(59). Esta última tampoco redujo la incidencia de FAPO en el estudio controlado randomizado STICS $^{(60)}$, además de asociarse a una tasa aumentada de injuria renal posoperatoria, por lo que pueden existir diferencias específicas entre los integrantes del grupo.

En otro metaanálisis, en este caso con diversas estatinas, tampoco se obtuvo protección contra la FAPO y se produjo una mayor incidencia de injuria renal aguda ${ }^{(61)}$.

Por lo tanto, luego de una visión inicial favorable, se ha generado un manto de duda en cuanto a la protección que las estatinas pudieran ofrecer sobre distintos eventos PO adversos, incluida la FAPO. Sin embargo, un amplio grupo de pacientes llega a cirugía bajo tratamiento con estatinas por otras indicaciones, y raramente se presenta una razón para suspenderlas. 


\section{Magnesio}

La baja concentración plasmática de este catión es predictor independiente de FAPO, aunque la suplementación I/V profiláctica de magnesio muestra resultados contradictorios. Un metaanálisis de Cochrane con 22 estudios pequeños o medianos, muy heterogéneos, mostró eficacia contra placebo ${ }^{(50)}$. Sin embargo, un posterior metaanálisis concluyó que los estudios pequeños originaban un sesgo hacia mejores resultados, mientras que analizando solo los estudios de mejor calidad no se mantenía el beneficio ${ }^{(62)}$. Además, el efecto del magnesio solo se observa sin el uso concomitante de betabloqueantes, y en su presencia no agregan protección ${ }^{(51)}$. Por lo tanto, el magnesio I/V podría ser una opción en presencia de contraindicación de betabloqueantes y amiodarona.

\section{Colchicina}

Es un potente antinflamatorio que actúa mediante la despolimerización de los microtúbulos.

El primer estudio randomizado sobre su papel en la profilaxis de la FAPO fue un subestudio del COPPS(63), donde se inició al tercer día del PO y se mantuvo durante un mes. La incidencia de FAPO a 30 días fue de $12 \%$ (colchicina) versus $22 \%$ (controles); $\mathrm{p}=0,021$, con -1 día de estadía hospitalaria ( $\mathrm{p}$ $=0,040$ ).

En el COPPS-2, la colchicina fue iniciada 48-72 h antes de la cirugía. Si bien se redujo el síndrome pospericardiotomía, no ocurrió igual con la FAPO, dada la frecuente suspensión de la colchicina por efectos gastrointestinales. Sin embargo, en el análisis por tratamiento preespecificado la reducción fue significativa ${ }^{(64)}$.

Varios metaanálisis apoyan la eficacia de la colchicina en la profilaxis de la FAPO. Dos son destacables por referirse específicamente al POCC: el de Salih ${ }^{(65)}$ con seis estudios y 1.257 pacientes, aunque con un aumento significativo de los efectos colaterales, y el de Lemmerz ${ }^{(66)}$ con cinco estudios y 1.412 pacientes, que observó una reducción de 1,2 días de estadía hospitalaria.

La evidencia a favor de la eficacia de la colchicina parece robusta, aunque su utilidad está limitada por sus efectos gastrointestinales.

\section{Glucocorticoides}

Su papel en la profilaxis de la FAPO es al menos dudoso. Aunque algunos metaanálisis les asignan un beneficio a varios corticoides ${ }^{(67,68)}$, adolecen de importante heterogeneidad en cuanto a fármacos, dosis, vía y oportunidad. Dos estudios grandes randomizados doble ciego, el DECS ${ }^{(69)}$ con 4.494 pacientes y el SIRS ${ }^{(70)}$ con 7.507 pacientes, no demostraron beneficio significativo. Por lo tanto, los estudios de mayor calidad no dan apoyo claro a su uso, lo que se suma a sus posibles efectos metabólicos y los riesgos de infección y compromiso de la cicatrización.

\section{Suplementos de potasio}

La hipopotasemia es frecuente en pacientes que van a cirugía cardíaca, y se ha identificado como un factor favorecedor de FAPO, de modo que su suplemento es utilizado en muchos centros. Si bien es adecuada su reposición cuando su nivel plasmático es bajo, no está avalado su aporte sistemático, y la mejor evidencia muestra que un objetivo de potasemia de $4,5 \mathrm{mEq} / 1$ no disminuye la incidencia de FAPO comparada con un objetivo de $4,0 \mathrm{mEq} / \mathrm{l}^{(71)}$. Está en curso el estudio Tight-K ${ }^{(72)}$, que compara un objetivo de potasemia $\geq 3,6 \mathrm{vs.} \geq 4,5 \mathrm{mEq} / \mathrm{l}$.

Otros fármacos evaluados incluyen antioxidantes (N-acetilcisteína y ascorbato), antinflamatorios no esteroideos, calcioantagonistas, ácidos grasos polinsaturados y digoxina.

Estos grupos de fármacos no han mostrado utilidad consistente en la profilaxis de la FAPO y no son considerados en las guías ${ }^{(4,7,20,52,73)}$.

En la tabla 1 se resumen las recomendaciones de las sociedades científicas sobre la elección de fármacos para la profilaxis de la FAPO.

\section{Estrategias no farmacológicas}

La profilaxis de la FAPO requiere una estrategia integral, que además de las medidas farmacológicas descritas contemple sus mecanismos fisiopatológicos en el intra y posoperatorio ${ }^{(14)}$. Estas acciones complementarias van dirigidas a controlar y mantener varias constantes fisiológicas con fuerte impacto sobre el tono simpático, el estado inflamatorio y el daño auricular directo (figura 6).

La sobrestimulación auricular es capaz de reducir la incidencia de FA corrigiendo la bradicardia y la carga de latidos ectópicos. En el metaanálisis de Cochrane de 22 estudios con importante heterogeneidad, redujo la FAPO significativamente ${ }^{(50)}$. La estimulación biatrial parece ser más efectiva que la de una aurícula, y se ha asociado a disminución de la internación ${ }^{(51)}$. Otros estudios no mostraron ventajas comparativas con respecto a metoprolol o amiodarona. Como limitaciones, no siempre se posicionan electrodos auriculares transitorios en la cirugía y no son raros los defectos de sensado y/o captura, además de los posibles riesgos de su remoción.

Otras estrategias no farmacológicas han mostrado beneficio en algunos estudios, aunque no han conseguido evidencia sólida ni consideración en las guías. La pericardiotomía posterior, al minimizar la reten- 


\begin{tabular}{|c|c|c|c|}
\hline Fármaco & Clase & Sociedades & Indicación condicional \\
\hline Betabloqueantes & I A & Todas & \\
\hline \multirow[t]{2}{*}{ Amiodarona } & I A & ESC 2020 & \\
\hline & $\mathrm{IIa}$ & El resto* & $\begin{array}{c}\text { contraindicados } \\
\text { Adicional a BB en alto riesgo de } \\
\text { FAPO }\end{array}$ \\
\hline \multirow[t]{2}{*}{ Sotalol } & $\mathrm{IIb}$ & AHA/ACC/HRS & \\
\hline & - & CCS & Alto riesgo de FAPO \\
\hline Colchicina & $\mathrm{IIb}$ & AHA/ACC/HRS & \\
\hline Magnesio & - & CCS & $\begin{array}{l}\text { Contraindicación de BB y } \\
\text { amiodarona } \\
\text { Adicional en alto riesgo }\end{array}$ \\
\hline Estimulación biauricular & - & CCS - EACTS & $\begin{array}{c}\text { Contraindicación de BB y } \\
\text { amiodarona }\end{array}$ \\
\hline
\end{tabular}

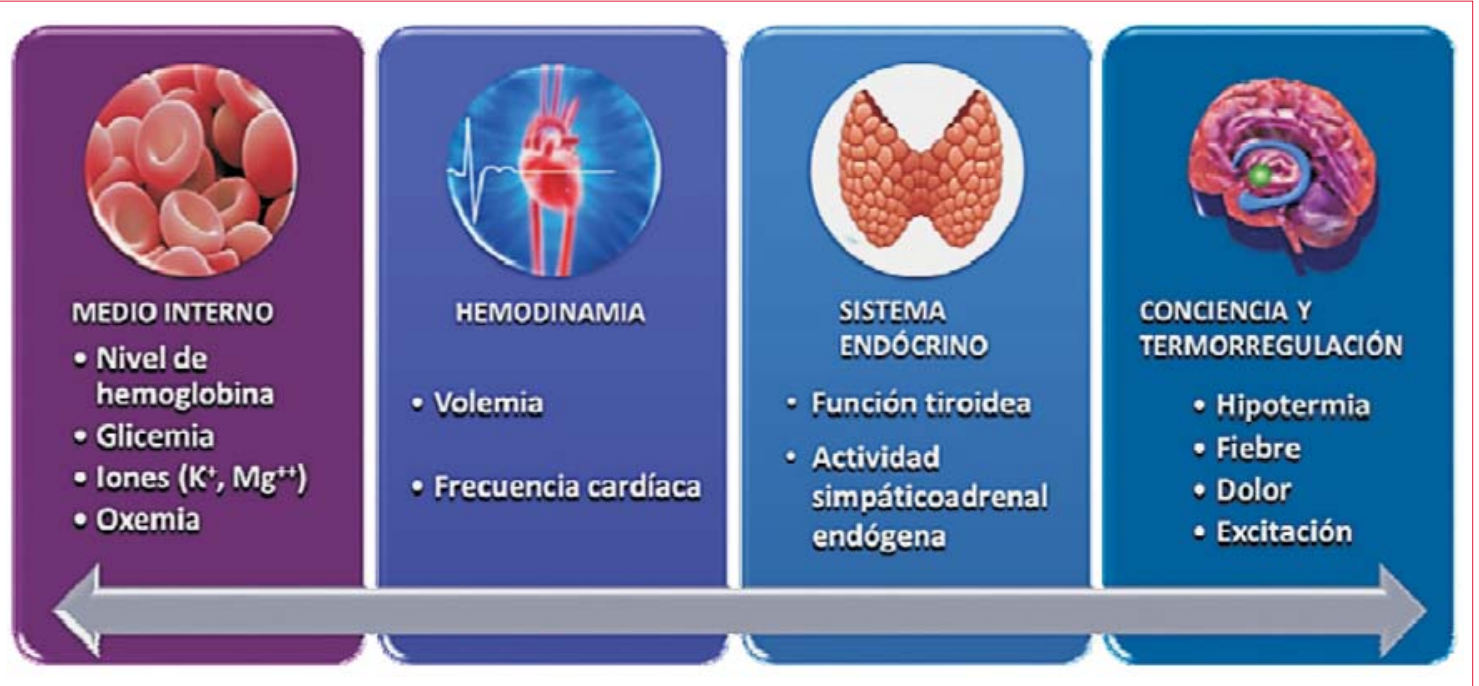

Figura 6. Profilaxis no farmacológica de la fibrilación auricular posoperatoria. Alteraciones en distintos sistemas fisiológicos participan en los mecanismos de inicio y mantenimiento de la arritmia. Las correcciones de estas variables constituyen un coadyuvante válido y necesario tanto en su profilaxis como en su tratamiento.

ción de fluido pericárdico, es capaz de disminuir el componente inflamatorio local y reducir la incidencia de derrame clínicamente relevante y de $\mathrm{FAPO}^{(52,73)}$.

También se ha propuesto cirugía coronaria sin CEC, fundamentada en la supresión de un gran activador de la respuesta inflamatoria sistémica, pero los estudios y metaanálisis no ofrecen resultados concluyentes, en especial en aquellos de mejor calidad y en los que el riesgo de influencia de la industria es bajo ${ }^{(4,74)}$.

\section{Tratamiento}

La FAPO a menudo es breve o fugaz, y puede no necesitar tratamiento alguno. Requiere intervención cuando genera efectos hemodinámicos adversos o cuando la persistencia o reiteración de la arritmia hacen prever un riesgo tromboembólico significativo. Además, traduce un grado variable de miopatía auricular con posibles consecuencias sobre el ritmo cardíaco y complicaciones futuras. El tratamiento 


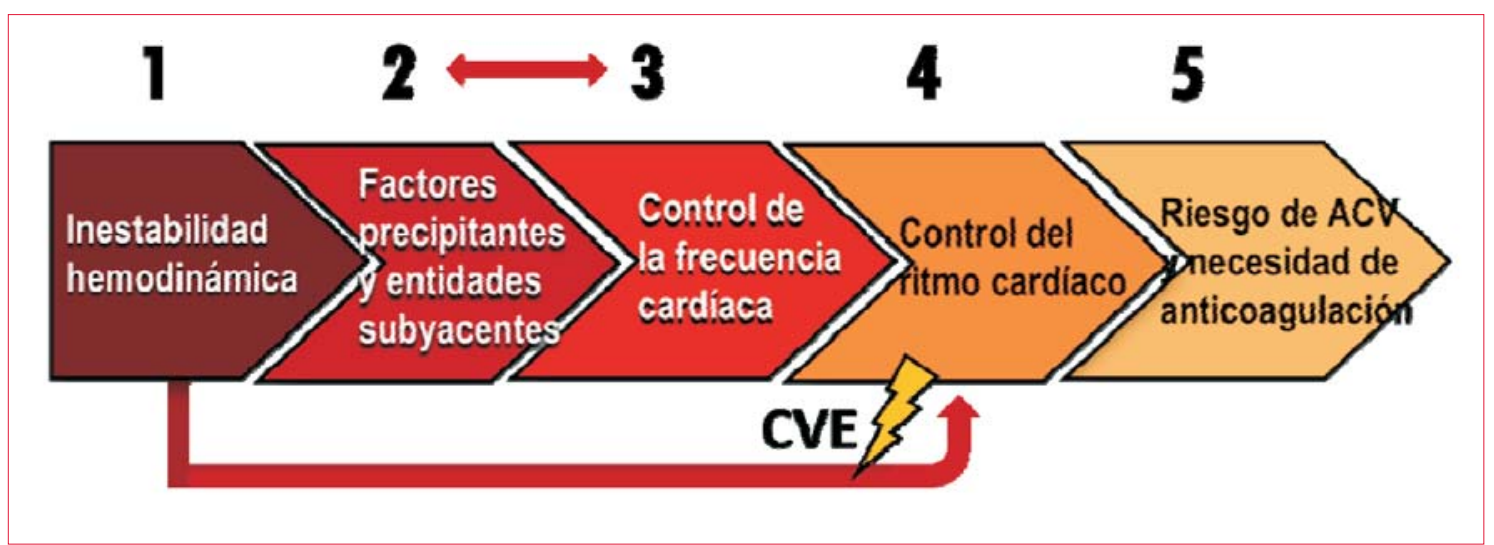

Figura 7. Orden de prioridades en el manejo de la fibrilación auricular posoperatoria. La inestabilidad hemodinámica requiere CVE inmediata. En la fibrilación auricular bien tolerada, la estrategia primaria recomendada por la ESC es el control de la frecuencia cardíaca ${ }^{(49)}$, aunque el control del ritmo es igualmente válido para la CCS ${ }^{(47)}$. Los factores precipitantes deben corregirse concomitantemente, cualquiera sea la conducta elegida. CVE: cardioversión eléctrica; ESC: European Society of Cardiology; CCS: Canadian Cardiovascular Society.

de la FA requiere contemplar cinco aspectos básicos con un orden de prioridad temporal (figura 7).

Lo primero será una rápida evaluación del estado hemodinámico y sintomático del paciente. Si se presentan signos de isquemia, hipotensión arterial o ICC se procede a la cardioversión eléctrica (CVE) sincronizada inmediata, que resulta altamente efectiva. La administración simultánea de antiarrítmicos I/V puede facilitar y estabilizar la conversión a ritmo sinusal, y ocasionalmente lograr la reversión mientras se prepara la CVE, o al menos un descenso de la frecuencia cardíaca (FC). La identificación clara del inicio de la arritmia hace innecesarios a la anticoagulación previa y el ecocardiograma transesofágico (ETE).

Si se presentan síntomas menos severos, puede intentarse cardioversión farmacológica (CVF) o proceder a la CVE si aquella fracasa. La FA de las primeras horas del PO con el paciente ventilado también se beneficia de la CVE, capitalizando la sedación en curso.

La refractariedad o rápida recurrencia de la arritmia pueden deberse a condiciones asociadas frecuentes en el POCC que siempre hay que identificar y corregir. Estas condiciones pueden tener un impacto propio y un tratamiento específico (derrame pericárdico, infección, anemia, hipoxemia, alteraciones de la volemia, isquemia, disfunción tiroidea, etcétera).

En la FA asintomática, las dos estrategias posibles son el control del ritmo cardíaco (CRC), o el control de la FC. Un estudio randomizado reciente no demostró diferencias en obtención de ritmo sinusal a los 60 días ni en la incidencia de ACV u otros eventos mayores, aunque hubo un cruzamiento considerable entre los grupos ${ }^{(75)}$.

La guía canadiense ${ }^{(47)}$ y la de la AHA/ACC/ $\mathrm{HRS}^{(48)}$ consideran razonables las dos estrategias, mientras que la ESC 2020 asigna preferencia (lla) al control de FC por analogía con la FA primaria ${ }^{(49)}$.

En el paciente con disfunción o hipertrofia ventricular severa, la recuperación aún transitoria de la actividad auricular puede proveer un mejor estado hemodinámico y funcional en el período más crítico de recuperación. Además teóricamente, limitar la carga precoz de FA y de remodelación atrial podría evitar formas más estables de la arritmia y anticoagulación prolongada, en especial si existe riesgo alto de sangrado. Por otra parte, la dificultad en lograr una FC adecuada obliga a reorientar la estrategia hacia el control del ritmo. El tratamiento de los factores precipitantes debe ser concomitante con cualquiera de estas estrategias.

\section{Control de la frecuencia cardíaca}

Varios agentes que reducen la conducción aurículoventricular son de uso habitual. El objetivo es una FC en reposo < 90 o 110 lpm, según las condiciones hemodinámicas del paciente. Los betabloqueantes son la primera elección, dado el elevado tono catecolaminérgico del PO, y deberían estar previamente incorporados por su papel preventivo. Los calcioantagonistas no dihidropiridínicos son la segunda opción; solo tienen prioridad si los betabloqueantes están contraindicados, y también pueden ser combinados con ellos.

Los digitálicos son de tercera elección, por ser menos efectivos en contexto de hipersimpaticotonía. Sin embargo, combinados con alguno de los anteriores pueden permitir reducir su dosis y sus efectos colaterales. Están contraindicados en presencia de una vía accesoria.

La cardiopatía de fondo también puede incidir fuertemente en la elección (figura 8). 


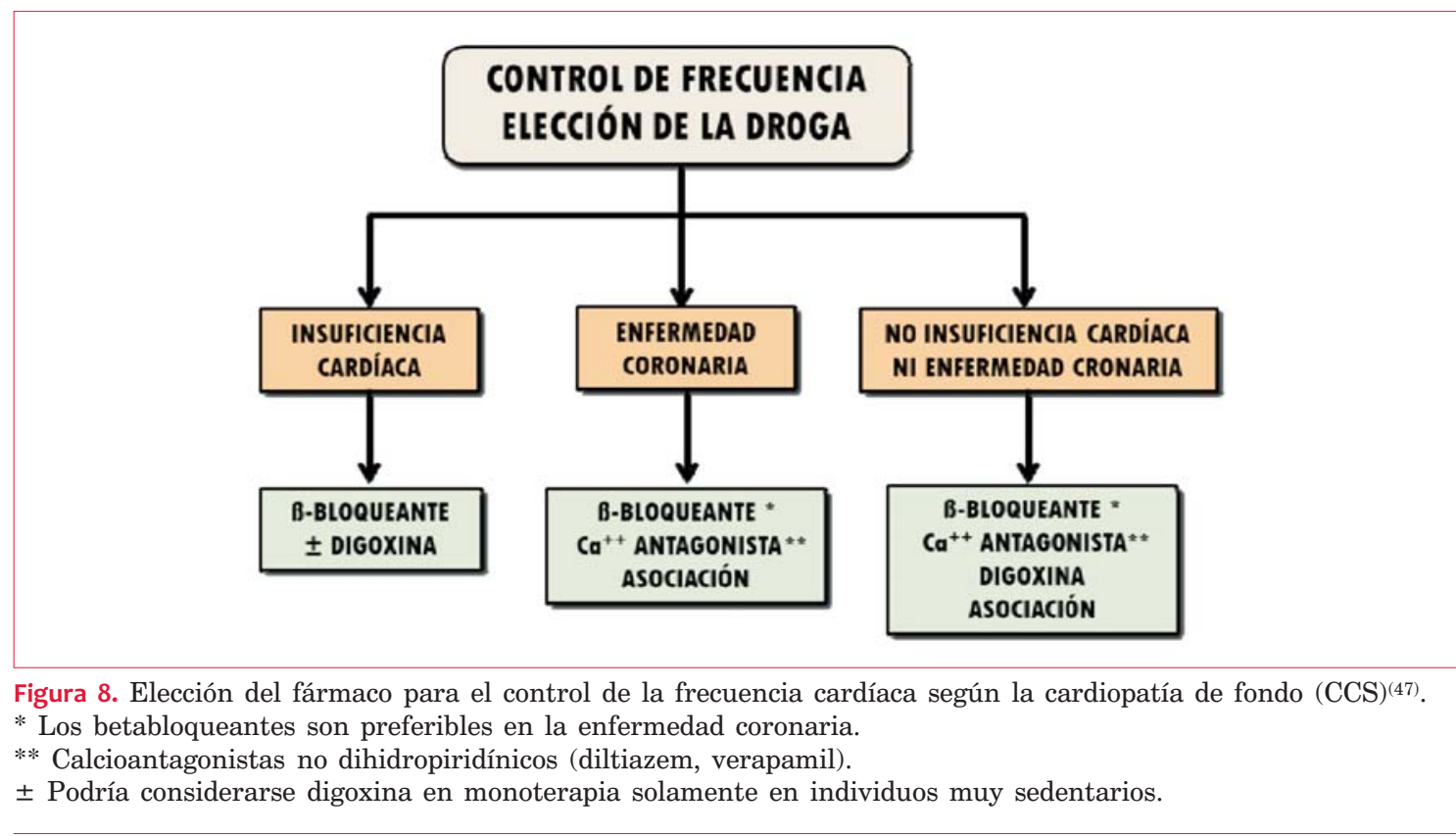

El control de FC requiere asociar anticoagulación inmediata y seguimiento del ritmo cardíaco para decidir el momento de su interrupción.

\section{Control del ritmo cardíaco en el paciente estable}

Si la estrategia elegida es el CRC, la administración del antiarrítmico seguirá de inmediato al diagnóstico, procurando evitar la remodelación y la necesidad de anticoagulación. Las recomendaciones se basan en la información obtenida en la FA primaria, dada la escasez de estudios específicos. Varias drogas son efectivas, pero la presencia de cardiopatía estructural, (coronariopatía, disfunción o hipertrofia ventricular) limita o contraindica el uso de drogas de los grupos la o lc en el POCC. La amiodarona $\mathrm{I} / \mathrm{V}$ es efectiva en la conversión y el mantenimiento del ritmo sinusal, tiene menor potencial proarrítmico que otros fármacos del grupo III y su toxicidad mayor se presenta en el mediano y largo plazo, por lo cual es la droga de elección en nuestro medio (tabla 2).

Se inicia con $5 \mathrm{mg} / \mathrm{kg} \mathrm{I} / \mathrm{V}$ en $250 \mathrm{ml}$ de suero glucosado al $5 \%$ en 15 a 20 minutos, seguida de una perfusión continua hasta completar una carga de 900 a $1.200 \mathrm{mg} / 24 \mathrm{~h}$. Luego de la reversión a ritmo sinusal se continúa por V/O como prevención secundaria, manteniendo los betabloqueantes de no existir contraindicación.

Si la arritmia persiste $>48 \mathrm{~h}$ sin anticoagulación, es recurrente o tiene inicio incierto se requiere un ETE para descartar trombosis cavitaria previo a la cardioversión, o diferirla tres semanas bajo anti- coagulación efectiva con INR entre 2,0 y 3,0 , suspendiendo los antiarrítmicos.

Luego de cardioversión exitosa se continuará con anticoagulación durante un plazo no menor a cuatro semanas y vigilancia estrecha del ritmo cardíaco. En un pequeño estudio no randomizado se observó que el tratamiento con betabloqueantes solos o asociados a amiodarona fue más eficaz que amiodarona aislada para evitar la recidiva de FA a los 30 días $^{(26)}$.

\section{Anticoagulación}

El siguiente pero no menos importante aspecto es la valoración del riesgo tromboembólico, determinado por la persistencia o reiteración de la arritmia y predictores integrados en el score $\mathrm{CHA}_{2} \mathrm{DS}_{2}$-VASc, aunque no validado en este contexto ${ }^{(73)}$. No hay un límite temporal seguro con respecto a este riesgo, pero la mayoría de las guías recomiendan indicar anticoagulación a las $24 \mathrm{o} 48 \mathrm{~h}$ de iniciada la FA. Por otra parte, el POCC se asocia a riesgo de sangrado por alteraciones de la hemostasis/fibrinolisis y presencia de suturas vasculares, por lo que la decisión y oportunidad de anticoagular debe condicionarse a un preciso balance, utilizando también el score HAS-BLED ${ }^{(76)}$.

Con FA $>24$ h o dos o más episodios, $\mathrm{CHA}_{2} \mathrm{DS}_{2-}$ VASc $\geq 2$ en el hombre $y \geq 3$ en la mujer y bajo o moderado riesgo de sangrado, debe iniciarse anticoagulación con heparina de bajo peso molecular (HBPM) simultáneamente con warfarina, con objetivo de INR entre 2,0 y 3,0 .

Una alternativa atractiva en la FAPO no valvular (ausencia de prótesis mecánica y de estenosis 


\begin{tabular}{|c|c|c|c|c|}
\hline \multirow[b]{2}{*}{ Prioridad } & \multicolumn{4}{|c|}{ Condición } \\
\hline & $\begin{array}{c}\text { Función ventricular } \\
\text { normal }\end{array}$ & $\begin{array}{c}\text { Disfunción ventricular } \\
\text { ICC }\end{array}$ & Coronariopatia & $\begin{array}{l}\text { Hipertrofia ventricular } \\
\text { izquierda }\end{array}$ \\
\hline $1^{\text {a }}$ elección & III: Amiodarona & III:Amiodarona & $\begin{array}{c}\text { III: Amiodarona } \\
\text { Sotalol }\end{array}$ & III:Amiodarona \\
\hline $2^{\mathrm{a}}$ elección & III: Sotalol & & & \\
\hline $3^{\mathrm{a}}$ elección & $\begin{array}{l}\text { Ia: Quinidina } \\
\text { Procainamidaæ } \\
\text { Disopiramida }\end{array}$ & & $\begin{array}{l}\text { Ia: Quinidina } \\
\text { Procainamida } \\
\text { Disopiramida }\end{array}$ & $\begin{array}{l}\text { Ia: Quinidina } \\
\text { Procainamida } \\
\text { Disopiramida }\end{array}$ \\
\hline Contraindicada & & $\begin{array}{l}\text { Ic: Flecainida } \\
\text { Propafenona }\end{array}$ & $\begin{array}{l}\text { Ic: Flecainida } \\
\text { Propafenona }\end{array}$ & $\begin{array}{l}\text { III: Sotalol } \\
\text { Ic: Flecainida } \\
\text { Propafenona }\end{array}$ \\
\hline
\end{tabular}

mitral moderada o severa) son los anticoagulantes directos, que no requieren puente de HBPM ni monitorización y compiten favorablemente en eficacia y seguridad con la warfarina ${ }^{(77)}$.

El fundamento de la anticoagulación precoz (< $24 \mathrm{~h}$ ) es permitir una CVE con bajo riesgo de complicaciones embólicas si la CVF o el control de FC fracasan, aunque en la práctica muchos profesionales no se sienten cómodos sin un ETE previo que descarte trombosis cavitaria.

La duración de la profilaxis tromboembólica luego de restablecido el ritmo sinusal es discutida; depende del estado de remodelación atrial prexistente y la carga de FA, que condicionan la posibilidad de recurrencia y el grado de disfunción mecánica pos-conversión.

Las guías recomiendan continuar al menos un mes, pero en caso de FA reiterada y/o evidencia de remodelación auricular significativa parece prudente prolongar el tratamiento y revaluar el ritmo cardíaco frecuentemente antes de su suspensión.

Una interesante comprobación es que el score $\mathrm{CHA}_{2} \mathrm{DS}_{2}$-VASc (integrado por varias condiciones asociadas a remodelación auricular) es predictor de $\mathrm{ACV}$ en forma independiente de la $\mathrm{FA}^{(78)}$ lo cual enfatiza la importancia de la miopatía atrial en esta complicación. En consecuencia, este score debería ser especialmente considerado en la decisión de suspender los anticoagulantes, más allá de la recuperación y aparente estabilidad del ritmo sinusal. De hecho, la actualización 2019 focalizada de la guía AHA/ACC/HRS 2014 emite una recomendación lla B-NR para anticoagulación prolongada luego de la $\mathrm{CV}$ de $\mathrm{FA} /$ Flutter $<48 \mathrm{~h}$ con $\mathrm{CHA}_{2} \mathrm{DS}_{2}$-VASc $\geq 2$ en el hombre $o \geq 3$ en la mujer, aunque no específicamente en el contexto quirúrgico ${ }^{(79)}$.
La nueva guía ESC 2020 propone como recomendación llb la anticoagulación a largo plazo, "considerando el beneficio neto anticipado y las preferencias del paciente"(49) (figura 9).

\section{Conclusiones}

La FAPO continúa siendo una complicación muy frecuente de la cirugía cardíaca a pesar de las numerosas medidas profilácticas propuestas, y es probable que esa realidad se mantenga en el futuro. Si bien la literatura sobre el tema es profusa, su estudio sistemático se ha dificultado debido a grandes inconsistencias en cuanto a definición, métodos de registro, población objetivo, diseño metodológico y manejo. No obstante, esta arritmia está siendo progresivamente contemplada no como un epifenómeno transitorio e inocuo de la cirugía, sino un evento asociado a graves complicaciones que incluyen ACV y mortalidad tanto en el perioperatorio como en el largo plazo, generando mayor uso de recursos y exceso de costos.

Por otra parte, los datos sólidos provenientes de estudios controlados randomizados son demasiado escasos como para asegurar que la reducción de su incidencia se traducirá efectivamente en un descenso de tales eventos adversos, por lo que buena parte de las recomendaciones de las guías para su manejo provienen de información obtenida de la FA ajena al contexto del perioperatorio. Este hecho y el temor a los efectos colaterales de los fármacos considerados de primera línea en su prevención explican la adherencia llamativamente baja en su aplicación.

Aun así, la información disponible sugiere fuertemente que la arritmia tiene una responsabilidad propia y directa en las complicaciones tromboembó- 


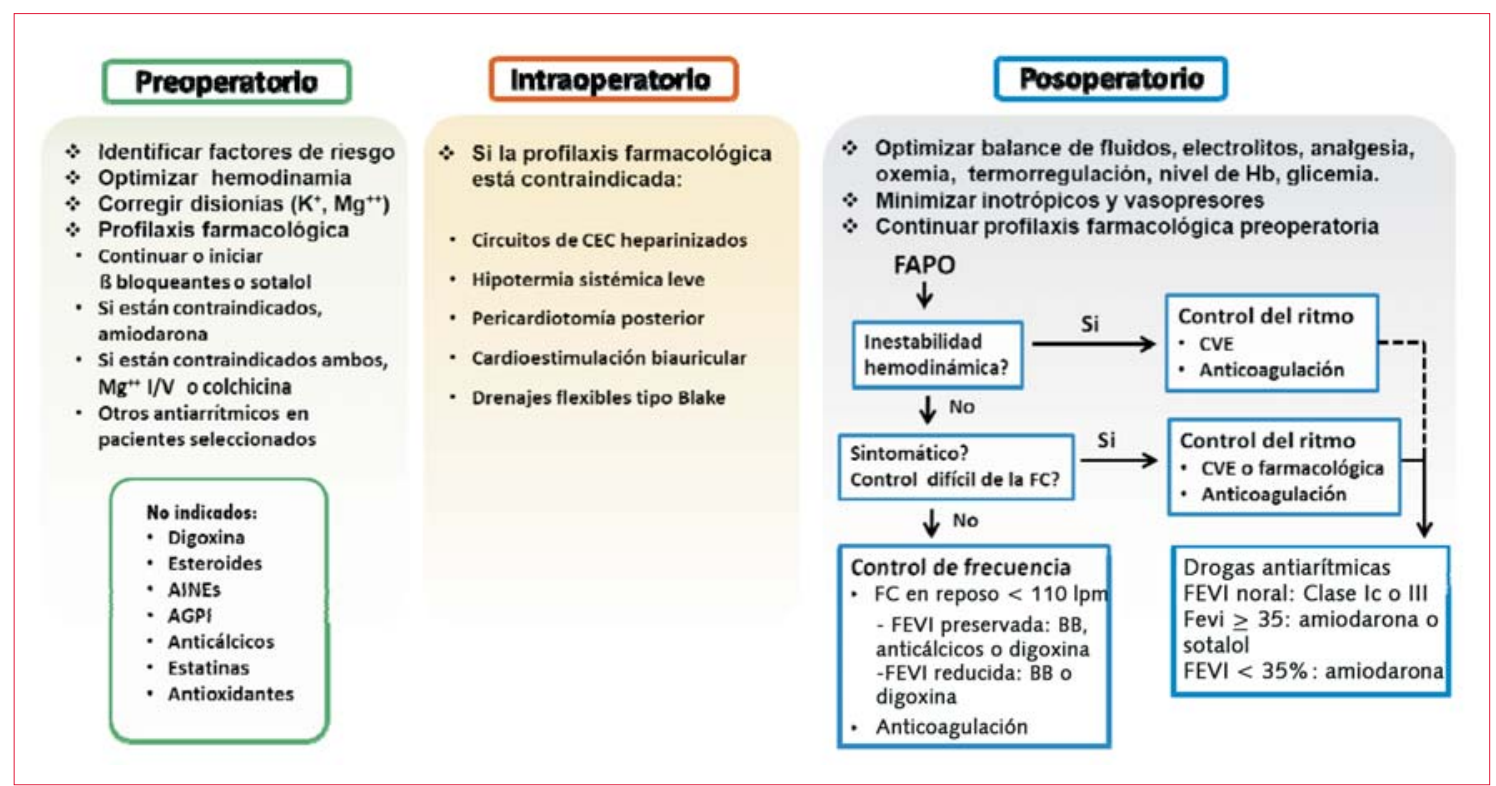

Figura 9. Principios básicos de la profilaxis y el manejo de la fibrilación auricular posoperatoria. AINEs: antinflamatorios no esteroideos; AGPI: ácidos grasos polinsaturados; CEC: circulación extracorpórea; Hb: Hemoglobina; CVE: cardioversión eléctrica; FC: frecuencia cardíaca; FEVI: fracción de eyección del ventrículo izquierdo. (Modificado de las guías ESC 2020 sobre fibrilación auricular $\left.{ }^{(49)}\right)$.

licas y la mortalidad a largo plazo, y que su irrupción en el contexto quirúrgico representa una manifestación de profundas modificaciones eléctricas y estructurales subclínicas del miocardio auricular, es decir de una verdadera miocardiopatía atrial de carácter progresivo.

Con esto en mente, la aparición de FAPO debería generar una alerta en el clínico responsable del seguimiento del paciente, que tendrá a su cargo la vigilancia del ritmo cardíaco, establecer un plan de prevención secundaria higiénico-farmacológico de la FA y sus comorbilidades relacionadas, y asumir decisiones tan importantes como definir el alcance temporal de la anticoagulación y la eventual necesidad y oportunidad de un tratamiento invasivo de la arritmia.

Estudios futuros bien diseñados podrían ayudar a definir mejor el valor de medidas preventivas concretas en la reducción de eventos mayores y obtener un modelo de estratificación de riesgo válido con el que racionalizar más apropiadamente su aplicación, minimizando tanto los riesgos de la arritmia como los derivados del uso indiscriminado de tales medidas. En el largo plazo, la interrogante a responder es si la implementación luego del alta de estrategias ya consolidadas en la FA primaria, como el seguimiento diligente del ritmo, la prevención secundaria y/o la anticoagulación ajustada al riesgo tromboembólico es capaz de mejorar el pronóstico de estos pacientes.

\section{Jorge Estigarribia Passaro,}

https://orcid.org/0000-0000-5136-7668

Este artículo fue aceptado para su publicación por: Editor jefe Dr. Gerardo Soca.

\section{Bibliografía}

1. Bessissow A, Khan J, Devereaux P, Álvarez-García J, Alonso-Coello P. Postoperative atrial fibrillation in non-cardiac and cardiac surgery: an overview. J Thromb Haemost. 2015; 13(Suppl 1): S304-12. doi: 10.1111/jth.12974

2. Shen J, Lall S, Zheng V, Buckley P, Damiano RJr, Schuessler R. The persistent problem of new-onset postoperative atrial fibrillation: a single-institution experience over two decades. J Thorac Cardiovasc Surg. 2011; 141(2):559-70. doi: 10.1016/ j.jtcvs.2010.03.011

3. Greenberg J, Lancaster T, Schuessler R, Melby S. Postoperative atrial fibrillation following cardiac surgery: a persistent complication. Eur J Cardiothorac Surg. 2017; 52(4):665-72. doi: 10.1093/ejcts/ ezx039

4. Omae T, Kanmura Y. Management of postoperative atrial fibrillation. J Anesth. 2012; 26(3):429-37. doi: 10.1007/s00540-012-1330-9

5. Cohn W, Gregoric I, Radovancevic B, Wolf R, Frazier O. Atrial fibrillation after cardiac transplantation: experience in 498 consecutive cases. Ann 
Thorac Surg. 2008; 85(1):56-8. doi: 10.1016/j.athoracsur.2007.07.037

6. Sattiraju S, Vats S, Krishnan B, K Kim S, Austin E, Can I, et al. Operative technique and atrial tachyarrhythmias after orthotopic heart transplantation. J Atr Fibrillation. 2012; 5(4):690. doi: 10.4022/jafib.690

7. Kaireviciute D, Aidietis A, Lip G. Atrial fibrillation following cardiac surgery: clinical features and preventative strategies. Eur Heart J. 2009; 30(4):410-25. doi: 10.1093/eurheartj/ehn609

8. Mathew J, Fontes M, Tudor I, Ramsay J, Duke $P$, Mazer C, et al; Investigators of the Ischemia Research and Education Foundation; Multicenter Study of Perioperative Ischemia Research Group. A multicenter risk index for atrial fibrillation after cardiac surgery. JAMA 2004 291(14):1720-9. doi: 10.1001/jama.291.14.1720

9. Leung J, Bellows W, Schiller N. Impairment of left atrial function predicts post-operative atrial fibrillation after coronary artery bypass graft surgery. Eur Heart J. 2004; 25(20):1836-44. doi: 10.1016/j.ehj. 2004.07.014

10. Zipes D. Atrial fibrillation: a tachycardia-induced atrial cardiomyopathy. Circulation 1997; 95(3):5624. doi: 10.1161/01.cir.95.3.562

11. Shen M, Arora R, Jalife J. Atrial Myopathy. JACC Basic Transl Sci. 2019; 4(5):640-54. doi: 10.1016/j. jacbts.2019.05.005

12. Maesen B, Nijs J, Maessen J, Allessie M, Schotten U. Post-operative atrial fibrillation: a maze of mechanisms. Europace. 2012; 14(2):159-74. doi: 10.1093/europace/eur208

13. Ahlsson A, Fengsrud E, Bodin L, Englund A. Postoperative atrial fibrillation in patients undergoing aortocoronary bypass surgery carries an eightfold risk of future atrial fibrillation and a doubled cardiovascular mortality. Eur J Cardiothorac Surg. 2010; 37(6):1353-9. doi: 10.1016/j.ejcts.2009.12.033

14. Chelazzi C, Villa G, De Gaudio A. Postoperative atrial fibrillation. ISRN Cardiol. 2011; 2011:203179. doi: 10.5402/2011/203179

15. Cameron M, Tran D, Abboud J, Newton E, Rashidian H, Dupuis J. Prospective External Validation of Three Preoperative Risk Scores for Prediction of New Onset Atrial Fibrillation After Cardiac Surgery. Anesth Analg. 2018; 126(1):33-8. doi: 10.1213/ANE.0000000000002112

16. Moe G, Rheinholdt W, Abildskow J. A computer model of atrial fibrillation. Am Heart J. 1964 67(2):200-20. doi: 10.1016/0002-8703(64)90371-0.

17. Haïssaguerre $\mathbf{M}$, Jaïs $\mathbf{P}$, Shah $\mathbf{D}$, Takahashi $\mathbf{A}$, Hocini M, Quiniou G, et al. Spontaneous initiation of atrial fibrillation by ectopic beats originating in the pulmonary veins. N Engl J Med. 1998; 339(10):659-66. doi: 10.1056/NEJM199809033391003

18. Nattel S, Dobrev D. Electrophysiological and molecular mechanisms of paroxysmal atrial fibrillation. Nat Rev Cardiol. 2016; 13(10):575-90. doi: 10.1038/nrcardio.2016.118

19. Creswell L, Schuessler R, Rosenbloom M, Cox J. Hazards of postoperative atrial arrhythmias. Ann Thorac Surg. 1993; 56(3):539-49. doi: 10.1016/0003-4975(93)90894-n

20. Jongnarangsin K, Oral H. Postoperative atrial fibrillation. Cardiol Clin. 2009; 27(1):69-78, viii. doi: 10.1016/j.ccl.2008.09.011

21. Almassi G, Schowalter T, Nicolosi A, Aggarwal A, Moritz T, Henderson W, et al. Atrial fibrillation after cardiac surgery: a major morbid event? Ann Surg. 1997; 226(4):501-11. doi: 10.1097/00000658-199710000-00011

22. Lotfi A, Wartak S, Sethi P, Garb J, Giugliano G. Postoperative atrial fibrillation is not associated with an increase risk of stroke or the type and number of grafts: a single-center retrospective analysis. Clin Cardiol. 2011; 34(12):787-90. doi: 10.1002/ clc. 21001

23. Tulla H, Hippeläinen M, Turpeinen A, Pitkänen O, Hartikainen J. New-onset atrial fibrillation at discharge in patients after coronary artery bypass surgery: short- and long-term morbidity and mortality. Eur J Cardiothorac Surg. 2015; 48(5):747-52. doi: 10.1093/ejcts/ezu526

24. Almassi G, Hawkins R, Bishawi M, Shroyer A, Hattler B, Quin J, et al; Veterans Affairs Randomized On/Off Bypass Follow-up Study (ROOBYFS) Group. New-onset postoperative atrial fibrillation impact on 5-year clinical outcomes and costs. J Thorac Cardiovasc Surg. 2019: S0022-5223(19) 32774-6. doi: 10.1016/j.jtcvs.2019.10.150

25. Loubani M, Hickey M, Spyt T, Galiñanes M. Residual atrial fibrillation and clinical consequences following postoperative supraventricular arrhythmias. Int J Cardiol. 2000; 74(2-3):125-32. doi: 10.1016/ s0167-5273(00)00229-1

26. Cioffi G, Cemin C, Russo T, Pellegrini A, Terrasi F, Ferrario G. Post-discharge recurrences of new-onset atrial fibrillation following cardiac surgery: impact of low-dose amiodarone and beta-blocker prophylaxis. Ital Heart J. 2000; 1(10):691-7.

27. Gialdini G, Nearing $\mathbf{K}$, Bhave $\mathbf{P}$, Bonuccelli $\mathbf{U}$, Iadecola C, Healey J, et al. Perioperative atrial fibrillation and the long-term risk of ischemic stroke. JAMA. 2014; 312(6):616-22. doi: 10.1001/jama.2014. 9143

28. Lowres N, Mulcahy G, Jin K, Gallagher R, Neubeck L, Freedman B. Incidence of postoperative atrial fibrillation recurrence in patients discharged in 
sinus rhythm after cardiac surgery: a systematic review and meta-analysis. Interact Cardiovasc Thorac Surg. 2018; 26(3):504-11. doi: 10.1093/icvts/ivx348

29. Lin M, Kamel H, Singer D, Wu Y, Lee M, Ovbiagele B. Perioperative/Postoperative Atrial Fibrillation and Risk of Subsequent Stroke and/or Mortality. Stroke. 2019; 50(6):1364-71. doi: 10.1161/STROKE AHA.118.023921

30. Megens M, Churilov L, Thijs V. New-Onset Atrial Fibrillation After Coronary Artery Bypass Graft and Long-Term Risk of Stroke: A Meta-Analysis. J Am Heart Assoc. 2017; 6(12):e007558. doi: 10.1161/ JAHA.117.007558

31. Kerwin M, Saado J, Pan J, Ailawadi G, Mazimba S, Salerno M, et al. New-onset atrial fibrillation and outcomes following isolated coronary artery bypass surgery: A systematic review and meta-analysis. Clin Cardiol. 2020; 43(9):928-34. doi: 10.1002/ clc. 23414

32. Stanley T, Mackensen G, Grocott H, White W, Blumenthal J, Laskowitz D, et al. The impact of postoperative atrial fibrillation on neurocognitive outcome after coronary artery bypass graft surgery. Anesth Analg. 2002; 94(2):290-5, table of contents. doi: 10.1097/00000539-200202000-00011

33. Villareal R, Hariharan R, Liu B, Kar B, Lee V, Elayda M, et al. Postoperative atrial fibrillation and mortality after coronary artery bypass surgery. J Am Coll Cardiol. 2004; 43(5):742-8. doi: 10.1016/j. jacc.2003.11.023

34. Kaw R, Hernández AV, Masood I, Gillinov A, Saliba W, Blackstone E. Short- and long-term mortality associated with new-onset atrial fibrillation after coronary artery bypass grafting: a systematic review and meta-analysis. J Thorac Cardiovasc Surg. 2011; 141(5):1305-12. doi: 10.1016/j.jtcvs. 2010.10 .040

35. El-Chami M, Kilgo P, Thourani V, Lattouf O, Delurgio D, Guyton R, et al. New-onset atrial fibrillation predicts long-term mortality after coronary artery bypass graft. J Am Coll Cardiol. 2010; 55(13):1370-6. doi: 10.1016/j.jacc.2009.10.058

36. Mariscalco G, Klersy C, Zanobini M, Banach $\mathbf{M}$, Ferrarese S, Borsani P, et al. Atrial fibrillation af ter isolated coronary surgery affects late survival. Circulation. 2008; 118(16):1612-8. doi: 10.1161/ CIRCULATIONAHA.108.777789

37. Thorén E, Hellgren L, Granath F, Hörte L, Ståhle E. Postoperative atrial fibrillation predicts cause-specific late mortality after coronary surgery. Scand Cardiovasc J. 2014; 48(2):71-8. doi: 10.3109/ 14017431.2014.880793

38. Wijffels M, Kirchhof C, Dorland R, Allessie M. Atrial fibrillation begets atrial fibrillation. A study in awake chronically instrumented goats. Circulation. 1995; 92(7):1954-68. doi: 10.1161/01.cir.92.7.1954
39. de Vos C, Pisters R, Nieuwlaat R, Prins M, Tieleman R, Coelen R, et al. Progression from paroxysmal to persistent atrial fibrillation clinical correlates and prognosis. J Am Coll Cardiol. 2010; 55(8):725-31. doi: 10.1016/j.jacc.2009.11.040

40. Shin H, Hashizume K, Iino Y, Koizumi K, Matayoshi T, Yozu R. Effects of atrial fibrillation on coronary artery bypass graft flow. Eur J Cardiothorac Surg. 2003; 23(2):175-8. doi: 10.1016/s1010-7940(02) 00730-3

41. Zhang Y, Mazgalev T. Atrioventricular node functional remodeling induced by atrial fibrillation. Heart Rhythm. 2012; 9(9):1419-25. doi: 10.1016/ j.hrthm.2012.04.019

42. Bramer S, ter Woorst F, van Geldorp M, van den Broek K, Maessen J, Berreklouw E, et al. Does new-onset postoperative atrial fibrillation after coronary artery bypass grafting affect postoperative quality of life? J Thorac Cardiovasc Surg. 2013; 146(1):114-8. doi: 10.1016/j.jtcvs.2012.06.045

43. Shirzad M, Karimi A, Tazik M, Aramin H, Ahmadi S, Davoodi S, et al. Factores determinantes de fibrilacíón auricular postoperatoria y el uso de recursos en cirugía cardíaca. Rev Esp Cardiol. 2010; 63(9):1054-60. doi: 10.1016/S0300-8932(10)70227-X

44. Kosuma P, Wachirasrisirikul S, Jedsadayanmata A. Attributable costs of postoperative atrial fibrillation among patients undergoing cardiac surgery. Cardiol Res Pract. 2018; 2018:3759238. doi: $10.1155 / 2018 / 3759238$

45. LaPar D, Speir A, Crosby I, Fonner EJr, Brown M, Rich J, et al; Investigators for the Virginia Cardiac Surgery Quality Initiative. Postoperative atrial fibrillation significantly increases mortality, hospital readmission, and hospital costs. Ann Thorac Surg. 2014; 98(2):527-33. doi: 10.1016/j.athoracsur.2014.03.039

46. Mitchell L; CCS Atrial Fibrillation Guidelines Committee. Canadian Cardiovascular Society atrial fibrillation guidelines 2010: prevention and treatment of atrial fibrillation following cardiac surgery. Can J Cardiol. 2011; 27(1):91-7. doi: 10.1016/ j.cjca.2010.11.005

47. Skanes A, Healey J, Cairns J, Dorian P, Gillis A, McMurtry M, et al; Canadian Cardiovascular Society Atrial Fibrillation Guidelines Committee. Focused 2012 update of the Canadian Cardiovascular Society atrial fibrillation guidelines: recommendations for stroke prevention and rate/rhythm control. Can J Cardiol. 2012; 28(2):12536. doi: 10.1016/j.cjca.2012.01.021

48. January C, Wann L, Alpert J, Calkins H, Cigarroa J, Cleveland JJr, et al; ACC/AHA Task Force Members. 2014 AHA/ACC/HRS guideline for the management of patients with atrial fibrillation: a report of the American College of Cardiology/Ameri- 
can Heart Association Task Force on practice guidelines and the Heart Rhythm Society. Circulation. 2014; 130(23):e199-267. doi: 10.1161/CIR.0000000000000041

49. Hindricks G, Potpara T, Dagres N, Arbelo E, Bax J, Blomström-Lundqvist C, et al; ESC Scientific Document Group. 2020 ESC Guidelines for the diagnosis and management of atrial fibrillation developed in collaboration with the European Association of Cardio-Thoracic Surgery (EACTS). Eur Heart J. 2020; ehaa612. doi: 10.1093/eurheartj/ehaa612

50. Arsenault K, Yusuf A, Crystal E, Healey J, Morillo C, Nair G, et al. Interventions for preventing post-operative atrial fibrillation in patients undergoing heart surgery. Cochrane Database Syst Rev. 2013; 2013(1):CD003611. doi: 10.1002/14651858. CD003611.pub3

51. Burgess D, Kilborn M, Keech A. Interventions for prevention of post-operative atrial fibrillation and its complications after cardiac surgery: a meta-analysis. Eur Heart J. 2006; 27(23):2846-57. doi: 10.1093/eurheartj/ehl272

52. Dobrev D, Aguilar M, Heijman J, Guichard J, Nattel S. Postoperative atrial fibrillation: mechanisms, manifestations and management. Nat Rev Cardiol. 2019; 16(7):417-36. doi: 10.1038/s41569 019-0166-5

53. Mitchell L, Exner D, Wyse D, Connolly C, Prystai G, Bayes A, et al. Prophylactic oral amiodarone for the prevention of arrhythmias that begin early after revascularization, valve replacement, or repair. PAPABEAR: a randomized controlled trial. JAMA. 2005; 294(24):3093-100. doi: 10.1001/jama. 294.24.3093

54. Buckley M, Nolan PJr, Slack M, Tisdale J, Hilleman D, Copeland J. Amiodarone prophylaxis for atrial fibrillation after cardiac surgery: meta-analysis of dose response and timing of initiation. Pharmacotherapy. 2007; 27(3):360-8. doi: 10.1592/phco.27.3.360

55. Irigoin J, Zócalo Y, Pouso J, Varela G, Bigalli D, Russo N, et al. Amiodarona como profilaxis de la fibrilación auricular en el postoperatorio de cirugía cardíaca. Rev Urug Cardiol. 2008; 23(2):134-41.

56. Osmanovic E, Ostojic M, Avdic S, Djedovic S, Delic A, Kadric N, et al. Pharmacological prophylaxis of atrial fibrillation after surgical myocardial revascularization. Med Arch. 2019; 73(1):19-22. doi 10.5455/medarh.2019.73.19-22

57. Patti G, Chello M, Candura D, Pasceri V, D'Ambrosio A, Covino E, et al. Randomized trial of atorvastatin for reduction of postoperative atrial fibrillation in patients undergoing cardiac surgery: results of the ARMYDA-3 (Atorvastatin for Reduction of MYocardial Dysrhythmia After cardiac sur- gery) study. Circulation. 2006; 114(14):1455-61. doi: 10.1161/CIRCULATIONAHA.106.621763

58. Kuhn E, Liakopoulos O, Stange S, Deppe A, Slottosch I, Choi Y, et al. Preoperative statin therapy in cardiac surgery: a meta-analysis of 90,000 patients. Eur J Cardiothorac Surg. 2014; 45(1):17-26. doi: $10.1093 /$ ejcts/ezt181

59. Sai C, Li J, Ruiyan M, Yingbin X. Atorvastatin prevents postoperative atrial fibrillation in patients undergoing cardiac surgery. Hellenic J Cardiol. 2019; 60(1):40-7. doi: 10.1016/j.hjc.2017.12.012

60. Zheng Z, Jayaram R, Jiang L, Emberson J, Zhao Y, Li Q, et al. Perioperative Rosuvastatin in Cardiac Surgery. N Engl J Med. 2016; 374(18): 1744-53. doi: 10.1056/NEJMoa1507750

61. Putzu A, Capelli B, Belletti A, Cassina T, Ferrari E, Gallo M, et al. Perioperative statin therapy in cardiac surgery: a meta-analysis of randomized controlled trials. Crit Care. 2016; 20(1):395. doi: 10.1186/s13054-016-1560-6

62. Cook R, Yamashita M, Kearns M, Ramanathan K, Gin K, Humphries K. Prophylactic magnesium does not prevent atrial fibrillation after cardiac surgery: a meta-analysis. Ann Thorac Surg. 2013; 95(2):533-41. doi: 10.1016/j.athoracsur.2012.09.008

63. Imazio M, Brucato A, Ferrazzi P, Rovere M, Gandino A, Cemin R, et al; COPPS Investigators. Colchicine reduces postoperative atrial fibrillation: results of the Colchicine for the Prevention of the Postpericardiotomy Syndrome (COPPS) atrial fibrillation substudy. Circulation. 2011; 124(21): 2290-5. doi: 10.1161/CIRCULATIONAHA.111.026153

64. Imazio M, Brucato A, Ferrazzi P, Pullara A, Adler Y, Barosi A, et al; COPPS-2 Investigators. Colchicine for prevention of postpericardiotomy syndrome and postoperative atrial fibrillation: the COPPS-2 randomized clinical trial. JAMA. 2014; 312(10):1016-23. doi: 10.1001/jama.2014.11026

65. Salih M, Smer A, Charnigo R, Ayan M, Darrat Y, Traina M, et al. Colchicine for prevention of post-cardiac procedure atrial fibrillation: Metaanalysis of randomized controlled trials. Int J Cardiol. 2017; 243:258-62. doi: 10.1016/j.ijcard.2017. 04.022

66. Lennerz C, Barman M, Tantawy M, Sopher M, Whittaker P. Colchicine for primary prevention of atrial fibrillation after open-heart surgery: systematic review and meta-analysis. Int J Cardiol. 2017; 249:127-37. doi: 10.1016/j.ijcard.2017.08.039

67. Viviano A, Kanagasabay R, Zakkar M. Is perioperative corticosteroid administration associated with a reduced incidence of postoperative atrial fibrillation in adult cardiac surgery? Interact Cardiovasc Thorac Surg. 2014; 18(2):225-9. doi: 10.1093/icvts/ ivt486 
68. Cappabianca G, Rotunno C, de Luca Tupputi Schinosa L, Ranieri V, Paparella D. Protective effects of steroids in cardiac surgery: a meta-analysis of randomized double-blind trials. J Cardiothorac Vasc Anesth. 2011; 25(1):156-65. doi: 10.1053/j.jvca. 2010.03.015

69. Dieleman J, Nierich A, Rosseel P, van der Maaten J, Hofland J, Diephuis J, et al; Dexamethasone for Cardiac Surgery (DECS) Study Group. Intraoperative high-dose dexamethasone for cardiac surgery: a randomized controlled trial. JAMA. 2012; 308(17):1761-7. doi: 10.1001/jama. 2012.14144

70. Whitlock R, Devereaux P, Teoh K, Lamy A, Vincent J, Pogue J, et al; SIRS Investigators. Methylprednisolone in patients undergoing cardiopulmonary bypass (SIRS): a randomised, double-blind, placebo-controlled trial. Lancet. 2015; 386(10000):1243-53. doi: 10.1016/S0140-6736(15) 00273-1

71. Hoekstra M, Hessels L, Rienstra M, Yeh L, Lansink A, Vogelzang M, et al. Computer-guided normal-low versus normal-high potassium control after cardiac surgery: No impact on atrial fibrillation or atrial flutter. Am Heart J. 2016; 172:45-52. doi: 10.1016/j.ahj.2015.10.020

72. Campbell N, Allen E, Sanders J, Swinson R, Birch S, Sturgess J, et al. The impact of maintaining serum potassium $=3.6 \mathrm{mEq} / \mathrm{L}$ vs $=4.5 \mathrm{mEq} / \mathrm{L}$ on the incidence of new-onset atrial fibrillation in the first 120 hours after isolated elective coronary artery bypass grafting - study protocol for a randomised feasibility trial for the proposed Tight $\mathrm{K}$ randomized non-inferiority trial. Trials. $2017 ; 18(1): 618$. doi: 10.1186/s13063-017-2349-x

73. Burrage P, Low Y, Campbell N, O'Brien B. New-Onset Atrial Fibrillation in Adult Patients After Cardiac Surgery. Curr Anesthesiol Rep. 2019; 9(2):174-93. doi: 10.1007/s40140-019-00321-4
74. Møller C, Penninga L, Wetterslev J, Steinbrüchel D, Gluud C. Off-pump versus on-pump coronary artery bypass grafting for ischaemic heart disease. Cochrane Database Syst Rev. 2012; (3):CD007224. doi: 10.1002/14651858.CD007224. pub2

75. Gillinov A, Bagiella E, Moskowitz A, Raiten J, Groh M, Bowdish M, et al. Rate control versus rhythm control for atrial fibrillation after cardiac surgery. N Engl J Med. 2016; 374(20):1911-21. doi: 10.1056/NEJMoa1602002

76. Pisters R, Lane D, Nieuwlaat $\mathbf{R}$, de Vos $\mathbf{C}$, Crijns H, Lip G. A novel user-friendly score (HAS-BLED) to assess 1-year risk of major bleeding in patients with atrial fibrillation: the Euro Heart Survey. Chest. 2010; 138(5):1093-100. doi: 10.1378/ chest.10-0134

77. Maan A, Padmanabhan R, Shaikh A, Mansour M, Ruskin J, Heist E. Newer anticoagulants in cardiovascular disease: a systematic review of the literature. Cardiol Rev. 2012; 20(5):209-21. doi: 10.1097/ CRD.0b013e3182503e2d

78. Peguero J, Issa O, Podesta C, Elmahdy H, Santana O, Lamas G. Usefulness of the $\mathrm{CHA}_{2} \mathrm{DS}_{2} \mathrm{VASc}$ score to predict postoperative stroke in patients having cardiac surgery independent of atrial fibrillation. Am J Cardiol. 2015; 115(6):758-62. doi: 10.1016/j.amjcard.2014.12.037

79. January C, Wann L, Calkins H, Chen L, Cigarroa J, Cleveland JJr, et al. 2019 AHA/ACC/HRS focused update of the $2014 \mathrm{AHA} / \mathrm{ACC} / \mathrm{HRS}$ guideline for the management of patients with atrial fibrillation: a report of the American College of Cardiology/American Heart Association Task Force on Clinical Practice Guidelines and the Heart Rhythm Society. J Am Coll Cardiol. 2019; 74(1):104-32. doi: 10.1016/j.jacc.2019.01.011 